\title{
New Applications of Synthetic Biology Tools for Cyanobacterial Metabolic Engineering
}

\author{
María Santos-Merino ${ }^{1 \dagger}$, Amit K. Singh ${ }^{1 \dagger}$ and Daniel C. Ducat ${ }^{1,2 *}$ \\ ${ }^{1}$ MSU-DOE Plant Research Laboratory, Michigan State University, East Lansing, MI, United States, ${ }^{2}$ Department of \\ Biochemistry and Molecular Biology, Michigan State University, East Lansing, MI, United States
}

Cyanobacteria are promising microorganisms for sustainable biotechnologies, yet unlocking their potential requires radical re-engineering and application of cutting-edge synthetic biology techniques. In recent years, the available devices and strategies for modifying cyanobacteria have been increasing, including advances in the design of genetic promoters, ribosome binding sites, riboswitches, reporter proteins, modular vector systems, and markerless selection systems. Because of these new toolkits, cyanobacteria have been successfully engineered to express heterologous pathways for the production of a wide variety of valuable compounds. Cyanobacterial strains with the potential to be used in real-world applications will require the refinement of genetic circuits used to express the heterologous pathways and development of accurate models that predict how these pathways can be best integrated into the larger cellular metabolic network. Herein, we review advances that have been made to translate synthetic biology tools into cyanobacterial model organisms and summarize experimental and in silico strategies that have been employed to increase their bioproduction potential. Despite the advances in synthetic biology and metabolic engineering during the last years, it is clear that still further improvements are required if cyanobacteria are to be competitive with heterotrophic microorganisms for the bioproduction of added-value compounds.

Keywords: cyanobacteria, metabolic engineering, synthetic biology, genome scale models, photosynthesis

${ }^{\dagger}$ These authors have contributed equally to this work

Specialty section: This article was submitted to

Synthetic Biology,

a section of the journal Frontiers in Bioengineering and

Biotechnology

Received: 30 November 2018 Accepted: 05 February 2019 Published: 27 February 2019

Citation:

Santos-Merino $M$, Singh AK and Ducat DC (2019) New Applications of Synthetic Biology Tools for Cyanobacterial Metabolic Engineering. Front. Bioeng. Biotechnol. 7:33. doi: 10.3389/fbioe.2019.00033

\section{INTRODUCTION}

Over 1 billion years ago, cyanobacteria began to drive the rise of oxygen in Earth's atmosphere, setting the stage for the evolution of new forms of complex, multicellular organisms (Shih, 2015). More recently, these photosynthetic prokaryotes have come under increasing scrutiny for their potential to sustain the lifestyle of complex eukaryotic lifeforms, this time as potential bioproduction hosts. Cyanobacteria can efficiently harvest $\mathrm{CO}_{2}$ as a carbon source, powering their metabolic processes by absorption of sunlight, the most abundant form of renewable energy. As fast-growing, and relatively simple bacteria, cyanobacteria hold the promise of being an ideal chassis for ambitious metabolic engineering projects. In recent years, cyanobacterial potential is being unlocked through the development of an increasing number of molecular tools (Berla et al., 2013; Carroll et al., 2018; Sun et al., 2018). These advances have been coupled with increasing capacities to manipulate endogenous genetic sequences and transfer exogenous DNA into multiple cyanobacterial strains (Berla et al., 2013; Cassier-Chauvat et al., 2016). Moreover, due to advances in sequencing technology, the genomes of $>270$ cyanobacterial species have been sequenced (Fujisawa et al., 2017), 
greatly facilitating the application of systems-level techniques, such as transcriptomics and proteomics. These technological improvements in the manipulation of cyanobacteria are set against the backdrop of increasingly refined tools that have been developed in the fields of metabolic engineering and synthetic biology, potentially setting the stage for cyanobacteria to significantly contribute as crop species in the Twentyfirst century.

Metabolic engineering can be defined as the practice of optimizing genetic and regulatory processes in cells to increase production of certain metabolic substances (Kumar and Prasad, 2011). This methodology has been used in cyanobacteria as well as other bacteria, to expand the list of products that they are able to make and increase production efficiency (Angermayr et al., 2015; Lai and Lan, 2015; Carroll et al., 2018). Traditional approaches to improve production of a specific compound were based on random mutagenesis and selection or the targeted introduction of individual genes and mutations, which requires considerable time to design and implement (Kumar and Prasad, 2011). Recently, systems metabolic engineering has emerged as new methodology for solving these issues (Nogales et al., 2013). It is based on the use of mathematic models to simulate and predict behaviors that emerge in complex systems, and has been used extensively for the improvement of microbial production (Lee et al., 2011).

In parallel, synthetic biology principles promote a bottom up approach to design biological systems, recombining defined parts or modules to restructure existing systems or build new pathways de novo (Sengupta et al., 2018). It is based on the construction of intricate biological systems using standardized, well-characterized, and interchangeable biological parts, or "modules" (Cheng and Lu, 2012). Collectively, these modules form "toolboxes" of components that can be used to modify an organism, including a catalog of characterized biological parts [e.g., promoters, ribosome binding sites (RBSs), riboswitches, terminator libraries], standardized methods for the assembly and manipulation of genetic components, and predictive models designed to facilitate pathway optimization (Gordon et al., 2016). Ideally, truly modular biological parts would be characterized in a manner that would allow researchers to accurately predict how they will function in the context of an interconnected system (Pasotti et al., 2012). In practice, it remains difficult to characterize parts in a manner that is completely transferrable to other organisms (Sengupta et al., 2018), because they are typically characterized by a specific research group in particular environment (Decoene et al., 2018). Another challenge to the transfer of parts is that biological components are usually non-orthogonal and may interact with genes, proteins, and metabolites of the chassis organism ( $\mathrm{Fu}, 2013)$, or have their function influenced by host pathway components (Wang et al., 2013). Still, largely by using specific parts and strategies developed in model heterotrophic organisms, useful synthetic biology components have been recently adapted and debugged for use in cyanobacteria.

This review focuses on recent engineering tools and strategies developed for metabolic engineering of cyanobacteria as hosts for generating added-value compounds while using solar energy and $\mathrm{CO}_{2}$ as inputs. We discuss the current challenges and opportunities for the application of synthetic biology principles in cyanobacteria. Finally, we highlight the potential for genomescale models as tools to assist cyanobacterial engineering.

\section{CYANOBACTERIA AS HOST FOR BIOMOLECULE PRODUCTION}

Cyanobacteria stand out as one of the most promising candidates as hosts for bioproduction (Knoot et al., 2018). Because cyanobacteria utilize solar energy to fix carbon dioxide, a greenhouse gas, and can convert these reduced carbon products into valuable metabolites (Lau et al., 2015), they are especially attractive in an era where sustainable biotechnological processes are of increasing importance (Ruffing, 2011). Additionally, cyanobacteria possess a number of advantageous features relative to other photosynthetic organisms. In comparison to eukaryotic algae and plants, cyanobacteria are more genetically tractable (Parmar et al., 2011; Lau et al., 2015), grow more rapidly, and can achieve higher efficiencies of solar energy capture and conversion (Dismukes et al., 2008; National Academies of Sciences, 2018). Furthermore, cyanobacteria can be cultivated without the need for arable landmass or potable water supplies (Nozzi et al., 2013) and can potentially even degrade aquatic pollutants, such as aromatic hydrocarbons (Ellis, 1977; Cerniglia et al., 1979, 1980; Narro et al., 1992) and xenobiotics (Megharaj et al., 1987; Kuritz and Wolk, 1995) to remediate contaminated water supplies. Yet, relative to other photosynthetic organisms, especially plants, cyanobacteria are currently not used in many scaled agricultural or biotechnological applications. The underutilization of cyanobacteria stems partially from their relative novelty as crop species. Whereas, technologies for cultivating, harvesting, and breeding plants have been under extensive development for many millennia, comparable research to improve the prospects for cyanobacterial cultivation has largely been pursued only since the 1970s (Sheehan et al., 1998).

Cyanobacteria make a number of compounds that are comparable to food, fiber, and fuel products routinely acquired from plants, although cyanobacterial strains have not been as extensively modified to improve their compatibility for scaled cultivation. Like many genera of eubacteria, cyanobacteria can synthesize polyhydroxyalkanoates, a thermoplastic class of biodegradable polyesters that includes polyhydroxybutyrate (Quintana et al., 2011). Many cyanobacterial strains also produce a wide spectrum of secondary metabolites with high-value commercial properties, such as pigments, vitamins, amino acids, macrolides, fatty acids, lipopeptides, and amides (Lau et al., 2015). In total, cyanobacteria are estimated to have the capacity to produce around 1,100 secondary metabolites (specific cyanobacterial bioproducts are beyond the scope of this article, but are reviewed comprehensively in Dittmann et al., 2015; Salvador-Reyes and Luesch, 2015; Xiong et al., 2015, 2017). Beyond natural metabolites, engineering efforts have been used to redirect the metabolism of model cyanobacteria toward biosynthesis of heterologous bioproducts including alcohols, fatty acids, hydrocarbons, fatty alcohols, olefins, organic 
acids, sugars, and polyols (engineered cyanobacterial metabolites reviewed in Lai and Lan, 2015; Knoot et al., 2018). Finally, biomass derived from cyanobacterial production processes could be used in animal feed supplements or converted into organic fertilizers, especially if cells are engineered for optimal nutritional and nutraceutical content (Singh et al., 2016).

Relative to heterotrophic microbial host species, cyanobacteria possess key limitations that have kept them from being as widely adopted as bioproduction chassis. First, in comparison to many heterotrophic workhorses, such as the bacterium Escherichia coli and the yeast Saccharomyces cerevisiae, cyanobacteria have relatively slow division rates. In part, this is due to the relative energy density that is contained in solar energy as compared to a rich medium (Zhang, 2011), since the carbohydrates and other organic carbon sources in rich media have high levels of potential energy stored in their bonds (Utschig et al., 2011). The low energy density of sunlight also impacts cyanobacterial autotrophic productivity, and the specific productivity of target metabolites tend to be lower than that of heterotrophic microbes (Nogales et al., 2013). Nevertheless, some species of cyanobacteria have division rates that compete with that of industrial yeasts ( $\mathrm{Yu}$ et al., 2015; Jaiswal et al., 2018) (see below). Secondly, the number of genetic tools available for cyanobacterial hosts continues to be limited relative to leading heterotrophic model organisms. Additionally, most cyanobacterial species are polyploid (Griese et al., 2011), which can complicate acquisition of fully segregated strains (Kelly et al., 2018), especially in the presence of restriction-modification systems in cyanobacteria that can limit transformation efficiencies (Stucken et al., 2013). Importantly, the technology for growing cyanobacteria at large scales is underdeveloped and low-cost bioreactors or other cultivation platforms systems such as open ponds need to be improved (Knoot et al., 2018). Bioreactors design must contend with the conflicting demands of scaling in two dimensions (to capture sunlight) while minimizing liquid volumes and reactor cost so that operational and capital expenses can be economically viable (Chisti, 2013; Nozzi et al., 2013; Acién et al., 2017). Genetic instability of heterologous pathways can also decrease bioproduction of cyanobacterial strains (Jones, 2014) and is increased by the abundance of repeated DNA motifs that lead to increased homologous recombination in cyanobacteria (CassierChauvat et al., 2016). As outlined below, a number of research efforts have been directed toward overcoming cyanobacterial host limitations in recent years.

\section{Model Cyanobacterial Strains}

While cyanobacteria are an extremely diverse phylum, a relatively small number of cyanobacterial strains have been selected as models, often because these strains have features that mitigate some of the limitations described above (Table 1). Notably, model workhorse cyanobacteria include Synechococcus elongatus PCC 7942 (hereafter S. elongatus PCC 7942), the first reported strain to be transformed through natural DNA uptake pathways (Shestakov and Khyen, 1970), and Synechocystis sp. PCC 6803 (Synechocystis PCC 6803), originally isolated in 1968 (Stanier et al., 1971), the first strain to have complex in silico models built for genome-scale prediction of metabolism (Fu, 2009). $S$. elongatus has been extensively used as a model of the circadian clock (Ditty et al., 2003), while Synechocystis PCC 6803 has served as a useful species for the investigation of core photosynthetic complexes due to its capacity to be grown under photoautotrophic, mixotrophic, or heterotrophic conditions (Vermaas, 1996). Nostoc sp. PCC 7120 (Nostoc PCC 7120) is a filamentous freshwater cyanobacterial strain which has been used extensively as a model to investigate cellular differentiation (Kumar et al., 2010). Nostoc PCC 7120 is capable of fixing nitrogen by forming heterocysts (Cai and Wolk, 1997), which are differentiated cells that efficiently catalyze the reduction of dinitrogen (Herrero et al., 2001), or be exploited for hydrogen production (Tamagnini et al., 2002). These three model species are arguably the best studied strains, but all have relatively modest doubling times: Nostoc PCC 7120 (14-15 h) (Callahan and Buikema, 2001), while S. elongatus PCC 7942 and Synechocystis PCC 6803 have doubling times around 7-12 h (Vermass et al., 1988; Mori et al., 1996). Furthermore, these established models have relatively limited capacity to withstand high light intensities and elevated temperatures that are expected to be encountered in outdoor bioreactors (Yu et al., 2013).

Driven by the desire for cyanobacterial models that are more amenable for bioindustrial applications, other cyanobacterial strains with much more rapid division times have emerged as important models. Significant efforts have been focused on Synechococcus sp. PCC 7002 (Synechococcus PCC 7002) in recent years, a unicellular cyanobacterium with a faster doubling time of $2.6 \mathrm{~h}$ (Ludwig and Bryant, 2012). Synechococcus PCC 7002 is also capable of growth in a variety of salt, temperature, and light conditions (Sheng et al., 2011; Ruffing et al., 2016), enabling the possibility of utilizing saltwater resources for growth media. More recently, still faster-growing strains have been reported, including Synechococcus elongatus UTEX 2973 (S. elongatus UTEX 2973) (Yu et al., 2015) and Synechococcus elongatus PCC 11801 (S. elongatus PCC 11801) (Jaiswal et al., 2018). Interestingly, although the growth rates of these strains are substantially faster $(1.5-3 \mathrm{~h})$, genome sequence and proteomic approaches have shown that they are exceptionally closelyrelated to the much slower-growing model, S. elongatus PCC 7942 (Mueller et al., 2017). Indeed, the genome of S. elongatus UTEX 2973 is $99.8 \%$ identical to S. elongatus PCC 7942 and differential regulation of a relatively small subset of common pathways largely accounts for the substantial growth differences (Abernathy et al., 2017; Mueller et al., 2017; Tan et al., 2018). Another related strain, S. elongatus PCC 11801 is 83\% identical to S. elongatus PCC 7942 and shares some key modifications with that of S. elongatus UTEX 2973 that are responsible for its higher growth rates and increased tolerance to certain environmental stresses (Jaiswal et al., 2018). In a recent publication, Ungerer et al. identified five single nucleotide polymorphisms (SNPs) in three genes (atpA, $p p n K$, and $r p a A)$ as responsible for rapid growth in S. elongatus UTEX 2973 (Ungerer et al., 2018) and these SNPs were also present in S. elongatus 11801. The atpA SNP yielded an ATP synthase with higher specific activity, the $p p n K$ SNP encoded a $\mathrm{NAD}^{+}$kinase with significantly improved kinetics, and the rpaA SNPs caused broad changes 
TABLE 1 | Common cyanobacterial model organisms.

\begin{tabular}{|c|c|c|c|c|}
\hline Strain & Genome size & Endogenous plasmids & Lifestyle features & DNA transfer methods \\
\hline Nostoc sp. PCC 7120 & $6.4 \mathrm{Mb}$ & $\begin{array}{l}6 \text { plasmids size ranging from } 5.6 \\
\text { to } 408 \mathrm{~kb}\end{array}$ & Freshwater; filamentous & $\begin{array}{l}\text { Conjugation (Wolk et al., 1984), electroporation } \\
\text { (Thiel and Poo, 1989) }\end{array}$ \\
\hline $\begin{array}{l}\text { Synechococcus } \\
\text { elongatus PCC } 7942\end{array}$ & $2.7 \mathrm{Mb}$ & $\begin{array}{l}46 \mathrm{~kb} \text { plasmid } \\
\text { ( } \& 7.6 \mathrm{~kb} \text { non-essential plasmid) }\end{array}$ & Freshwater; unicellular & $\begin{array}{l}\text { Conjugation (Tsinoremas et al., 1994), natural } \\
\text { transformation (Shestakov and Khyen, 1970), } \\
\text { electroporation (Marraccini et al., 1993) }\end{array}$ \\
\hline $\begin{array}{l}\text { Synechococcus } \\
\text { elongatus UTEX } 2973\end{array}$ & $2.7 \mathrm{Mb}$ & $46 \mathrm{~kb}$ plasmid & Freshwater; unicellular & Conjugation (Yu et al., 2015) \\
\hline $\begin{array}{l}\text { Synechococcus sp. } \\
\text { PCC } 7002\end{array}$ & $3.0 \mathrm{Mb}$ & $\begin{array}{l}6 \text { plasmids size ranging from } 4.8 \\
\text { to } 186 \mathrm{~kb}\end{array}$ & Euryhaline; unicellular & $\begin{array}{l}\text { Conjugation (Kopka et al., 2017), natural } \\
\text { transformation (Stevens and Porter, 1980) }\end{array}$ \\
\hline $\begin{array}{l}\text { Synechocystis sp. PCC } \\
6803\end{array}$ & $3.6 \mathrm{Mb}$ & $\begin{array}{l}7 \text { plasmids size ranging from } 2.3 \\
\text { to } 120 \mathrm{~kb}\end{array}$ & Freshwater; unicellular & $\begin{array}{l}\text { Conjugation (Marraccini et al., 1993), natural } \\
\text { transformation (Grigorieva and Shestakov, } \\
\text { 1982), ultrasonic transformation (Zang et al., } \\
\text { 2007), electroporation (Marraccini et al., 1993) }\end{array}$ \\
\hline
\end{tabular}

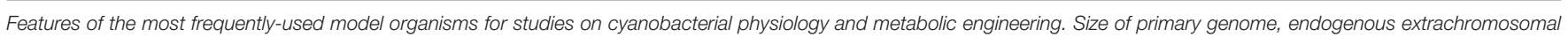
plasmids, osmotolerance, and routinely utilized genetic transformation methods are reported.

in the transcriptional profile. The filamentous cyanobacterium Leptolyngbya sp. strain BL0902 (Leptolyngbya BL0902) was also recently identified (Taton et al., 2012), can be transformed by conjugation (Taton et al., 2012) and some molecular tools have been characterized (Taton et al., 2014).

Curiously, while S. elongatus UTEX 2973 is closely related to S. elongatus PCC 7942, it can be only transformed by conjugation ( $\mathrm{Yu}$ et al., 2015), while S. elongatus PCC 11801 is naturally competent for genetic transformation. Apart from Nostoc PCC 7120, the other models described above are naturally competent, facilitating the genetic modification of these strains (Porter, 1986; Koksharova and Wolk, 2002). Furthermore, the genomes of the model cyanobacteria described in Table 1 have been sequenced and the information has been organized in the database, CyanoBase (Fujisawa et al., 2017). The access of genome information, together with the construction of metabolic models (see below), makes it possible to both understand the basic metabolism of cyanobacteria and to achieve higher levels of metabolic redirection and control (Lai and Lan, 2015).

\section{NEW TOOLS FOR SYNTHETIC BIOLOGY IN CYANOBACTERIA}

In comparison to the genetic toolboxes for work in popular heterotrophic chasses like E. coli and S. cerevisiae, relatively limited genetic tools have been developed in cyanobacteria (Sun et al., 2018). The design of standardized gene expression parts (e.g., promoters, terminators) that can be modularly recombined with other genetic elements with predictable outputs has catalyzed a revolution in the complexity of heterotrophic genetic circuits (Popp et al., 2017). Unfortunately, many of the synthetic biology tools and modular parts developed for model heterotrophs often do not perform as robustly in the context of cyanobacterial strains (Huang et al., 2010). For example, many characterized E. coli promoters do not display similar traits when used in cyanobacteria (Heidorn et al., 2011). These problems may arise in part because of species-dependent distinctions in key features, such as RBS sequences or promoter recognition by endogenous transcription, leading to unpredictability in gene expression (Huang et al., 2010; Wang et al., 2012; Camsund and Lindblad, 2014). In recent years, significant efforts have been made in adapting molecular techniques from other organisms in order to extend cyanobacterial toolboxes and facilitate cellular reprogramming for increased production yields. Here, we outline the latest advances in cyanobacterial synthetic biology tools, including promoters, riboswitches, RBSs, reporters, modular vectors, and markerless selection systems.

\section{Engineering Promoters to Enhance Protein Expression}

Many of the most advanced synthetic biology circuits and pathways today are firmly rooted in an extensive, well-defined library of promoter "parts" that exhibit predictable behaviors when used to drive the expression of a range of target genes (Shetty et al., 2008). By contrast, the number of constitutive and inducible promoters that have been well-characterized in cyanobacteria has been historically small, and considerable variation is often observed in the expression level achieved for distinct heterologous genes. Some limiting factors have compromised the development of cyanobacterial inducible genetic systems, including toxicity of inducers, leaky expression in the absence of inducer, and inducer photolability. Constitutive promoters may also be useful when continuous gene expression is desired, although many endogenous cyanobacterial promoters that are used for this purpose are dynamically regulated by circadian rhythms.

A few recent studies have focused on expanding and characterizing the collection of foreign promoters that can be used to drive gene expression in cyanobacteria. Some inducible promoter elements have been commonly used in selected cyanobacterial models for a number of years, such as the nickelinducible $n r s B$ promoter (Englund et al., 2016; Santos-Merino et al., 2018) or the IPTG-responsive $\operatorname{trc}$ promoter $\left(\mathrm{P}_{t r c}\right)$ in $S$. elongatus PCC 7942 or Synechocystis PCC 6803 (Geerts et al., 1995; Huang et al., 2010). $\mathrm{P}_{t r c}$ has recently been adapted to 
other model cyanobacteria, such as Synechococcus PCC 7002 (Ruffing, 2014) and Leptolyngbya BL0902 (Ma et al., 2014). The Larabinose-inducible araBAD promoter $\left(\mathrm{P}_{\mathrm{BAD}}\right)$ that has long been a staple induction system in E. coli has recently been introduced in S. elongatus PCC 7942 (Cao et al., 2017) and Synechocystis PCC 6803 (Immethun et al., 2017). This system is based on the arabinose utilization network, which positively regulates $\mathrm{P}_{\mathrm{BAD}}$ through the AraC regulator protein (Schleif, 2010) (Figure 1A). Most recently, the use of the rhamnose-inducible rhaBAD promoter of $E$. coli has been implemented in the model freshwater cyanobacterium Synechocystis PCC 6803 (Kelly et al., 2018) (Figure 1B). Another orthogonal inducible promoter, $P_{\text {van }}$ from Corynebacterium glutamicum, that relies upon vanillateinduced suppression of the repressor VanR has been tested in S. elongatus PCC 7942 (Taton et al., 2017) (Figure 1C). Some of these promoters have been translated to fastergrowing cyanobacterial strains, such as $\mathrm{P}_{\text {trc }}$ in Synechococcus PCC 7002 (Ruffing, 2014) and the IPTG-induced promoter $\mathrm{P}_{\text {lac }}$ in S. elongatus UTEX 2973 (Song et al., 2016), although the number of inducible promoters remains relatively limited in the cyanobacterial strains that possess the most promising features for bioproduction.

In some model cyanobacteria, a limited number of constitutive promoters have been characterized relative to one another. For example, $\mathrm{P}_{\mathrm{A} 2520}$ and $\mathrm{P}_{\mathrm{A} 2579}$, two native promoters of Synechococcus PCC 7002, have been found to drive strong expression of heterologous genes (Ruffing et al., 2016). Most recently, nine native promoters have been characterized for their expression in Synechocystis PCC 6803 to enrich the promoter toolboxes (Liu and Pakrasi, 2018). Other examples of publications that characterize endogenous promoters in cyanobacteria can be found here (Huang et al., 2010; Markley et al., 2015). Yet, in cyanobacteria, many endogenous promoters are strongly influenced by the circadian clock machinery, and therefore they may not be truly constitutive through a $24 \mathrm{~h}$ period (Liu et al., 1995; Markson et al., 2013; Camsund and Lindblad, 2014).

Development of synthetic promoters that have been modified for improved expression specifically in cyanobacteria has emerged as another promising strategy. In S. elongatus PCC 7942, a tandem promoter composed of a truncated native promoter PR from rrnA of S. elongatus PCC 7942, and the consensus- $\sigma 70$ PS promoter from $E$. coli has been designed (Chungjatupornchai and Fa-Aroonsawat, 2014). Zhou et al. reported the development of a constitutive promoter $\mathrm{P}_{c p c 560}$ in Synechocystis PCC 6803 that produced a high-level of gene expression (Zhou et al., 2014). It is based on a truncated native promoter $\left(\mathrm{P}_{c p c B}\right)$ and its strength resides in the presence of multiple transcription factor binding sites. In another study, $\mathrm{P}_{\text {sca3-2 }}$, a variant of $\mathrm{P}_{t a c}$ of $E$. coli was found to act as constitutive promoter with high levels of expression (Albers et al., 2015). Within the same context of synthetic constitutive promoters, a truncated version of $p s b A 2$ native promoter was developed in Synechocystis PCC 6803 (Englund et al., 2016). The expression of this derivate of $\mathrm{P}_{p s b A 2}$ increased 4-fold compared to the original. Similarly, inducible promoters can be tuned for better performance in cyanobacteria, such as the anhydrotetracycline-activated variant of $\mathrm{P}_{\mathrm{R} 40}$ from E. coli, $\mathrm{P}_{\mathrm{L} 03}$ (Huang and Lindblad, 2013), the IPTG-inducible promoter $\mathrm{P}_{\text {sca6-2 }}$ (Albers et al., 2015), or the T7 RNA Polymerase promoter (Ferreira et al., 2018). Because the promoters described above are modified and/or heterologous, it is possible that they could escape regulatory activities of the circadian clock, yet it is unusual for these promoters to be characterized in more than one circadian period, or at varied light intensities. To solve this problem, some promoters have been characterized for their capacity to drive heterologous gene expression under multiple conditions (e.g., light/dark, aerobic/anaerobic) such as the FNRactivated promoter, $\mathrm{P}_{\mathrm{O} 2}$, described in Synechocystis PCC 6803 (Immethun et al., 2016).

\section{Optimizing Ribosome Binding Sites for Biotechnological Applications}

The rate of protein production from a mRNA transcript also depends on the strength of the RBS in recruiting ribosomes for translation. The position and sequence of a given RBS significantly influences translational efficiency. Although "RBS calculators" have long been under development for heterotrophic microbes (Salis, 2011), it is only relatively recently that such efforts extended toward development of RBS libraries for cyanobacteria. RBS sequences from the BioBrick Registry of standard biological parts have been characterized in Synechocystis PCC 6803 (Heidorn et al., 2011; Englund et al., 2016). In one recent example, 20 native RBS elements have been characterized in Synechocystis PCC 6803 (Liu and Pakrasi, 2018), including two previously described by Englund et al. (2016). These efforts are becoming increasingly coordinated with attempts to develop synthetic RBSs that are based on in silico modeling tools for Synechococcus PCC 7002 (Markley et al., 2015), Synechocystis PCC 6803 (Heidorn et al., 2011; Taton et al., 2014; Xiong et al., 2015; Thiel et al., 2018), or S. elongatus PCC 7942 (Taton et al., 2014); (Wang et al., 2018).

Efforts to expand the toolbox of characterized promoter elements are foundational contributions that will enable more sophisticated circuit design in cyanobacteria, yet the capacity to fully predict expression output from a given element remains elusive. Context-specific features of a given expression construct can alter the performance of a given promoter-RBS combination, decreasing the modularity of promoter elements. For example, it is well-established that secondary structure can arise between a specific heterologous gene that can interfere with transcription or translation, leading to variability when expressing different genes from an identical promoter-RBS cassette. Promoter elements that are based on a bicistronic design have been developed for E. coli that exhibit much more consistent performance, regardless of the downstream gene sequence that is being expressed (Mutalik et al., 2013). No such system has been described in cyanobacteria and few promoters have been as extensively characterized. Therefore, it often remains difficult to anticipate the likely expression level of a construct during the design phase. 

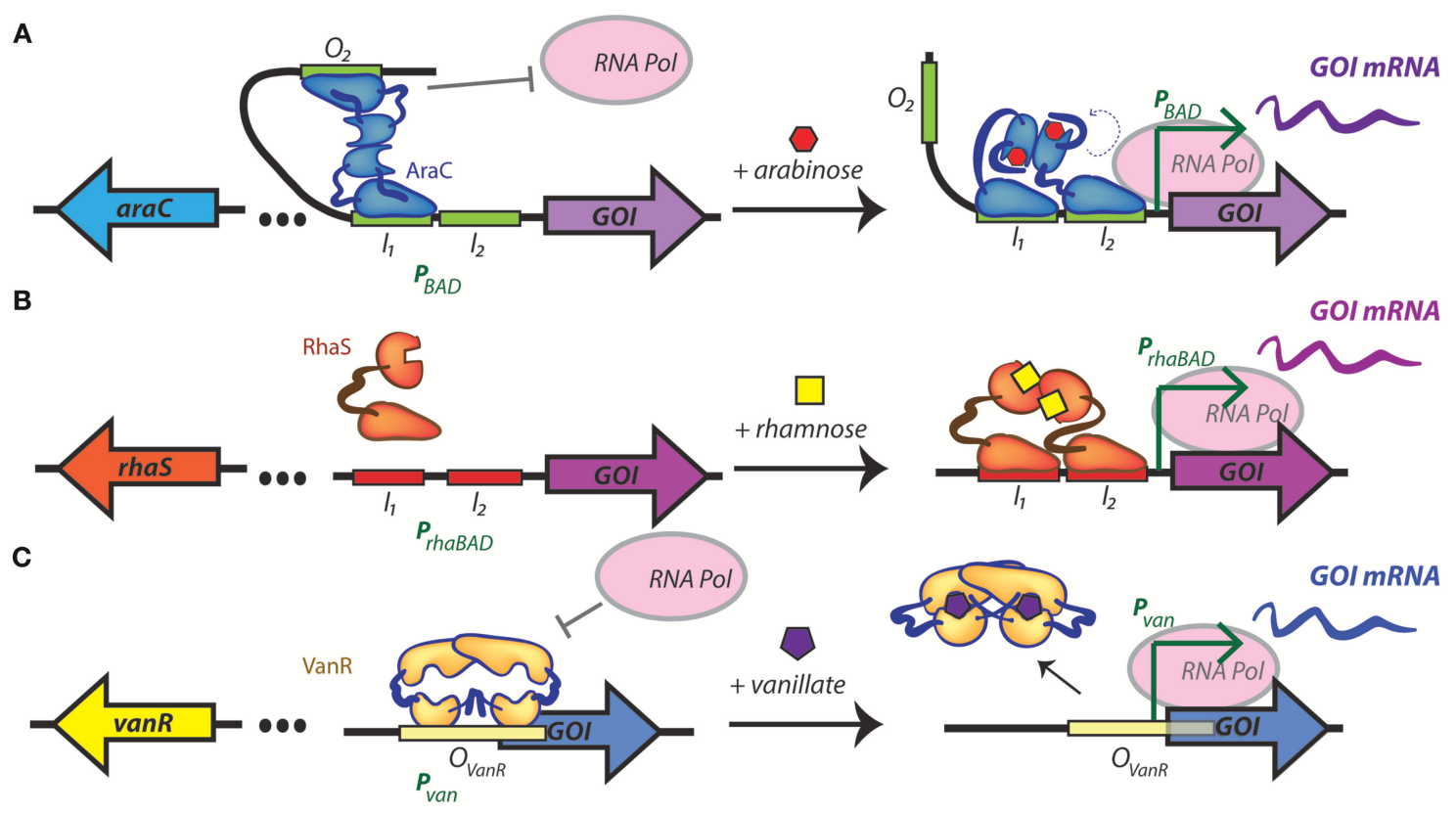

FIGURE 1 | Regulatory mechanisms of inducible promoters recently introduced into cyanobacteria. (A) Arabinose-inducible gene expression from $\mathrm{P}_{\mathrm{BAD}}$, which is both positively and negatively regulated by the transcriptional regulator AraC. In the absence of arabinose, an AraC dimer contacts the $\mathrm{O}_{2}$ and $\mathrm{I}_{1}$ half sites of the promoter favoring the formation of a DNA loop that is unfavorable for RNA polymerase binding. When arabinose is present, AraC undergoes a structural change that releases the DNA loop, and ideally positions an AraC activation domain that promotes gene transcription. (B) Rhamnose-inducible gene expression from $\mathrm{P}_{\text {rhaBAD. }}$ In the absence of rhamnose RhaS is unable to dimerize, limiting its capacity to bind to promoter elements. In the presence of rhamnose, a dimer of RhaS binds to the $\mathrm{I}_{1}$ and $\mathrm{I}_{2}$ repeat half-sites of the rhaBAD promoter, recruiting RNA polymerase and activating the transcription of the target gene. (C) The vanillate-inducible promoter, $\mathrm{P}_{\text {van }}$, is repressed by a dimer of VanR bound to its operator. When vanillate is present, two molecules bind the VanR dimer, resulting in a conformation change and release of transcriptional repression. $\mathrm{I}_{1}$, binding site 1 in $\mathrm{P}_{\mathrm{BAD}}$ and $\mathrm{P}_{\text {rhaBAD }}$; $\mathrm{l}_{2}$, binding site 2 in $\mathrm{P}_{\mathrm{BAD}}$ and $\mathrm{P}_{\text {rhaBAD }}$; GOI, gene of interest; mRNA, messenger RNA; $\mathrm{O}_{2}$, operator of $\mathrm{P}_{\mathrm{BAD}} ; \mathrm{O}_{\mathrm{vanR}}$, operator in $\mathrm{P}_{\text {van }}$; RNA pol, RNA polymerase. In all cases, left and right panel represent uninduced and induced promoters, respectively.

\section{Riboswitches as Tools for Robust Control of Gene Expression}

Riboswitches are versatile tools for genetic engineering and allow control of gene expression by manipulating secondary structure within a mRNA transcript. A riboswitch is composed of an aptamer sequence that imposes a secondary-structural conformation on the mRNA and which influences translational efficiency from the transcript. The aptamer sequence has cisactivating or cis-repressing effects on the mRNA on which it is encoded, while a trans-acting factor can bind and encourage the formation of an alternative secondary-structure conformation (Nudler, 2006). Frequently, the aptamer sequence is designed to form a stem-loop structure that prevents the attachment of ribosomes to a $5^{\prime}$ RBS. A trans-acting factor (which can be a small molecule metabolite or a non-coding regulatory RNA) binds to the aptamer in a manner that stimulates a new conformational state and increases accessibility of the RBS, improving translation of the encoded protein. Riboregulators have features that make them powerful tools for controlling gene expression when used in tandem with transcriptional-based approaches. These include the fact that they typically exhibit a high degree of modularity, can drive the expression of proteins over a large, physiologically-relevant range (thus avoiding issues such as toxicity or inclusion body formation), minimize "leaky" expression (protein production in the absence of inductor), have fast response times (time between ligand binding and protein expression), are tunable (a large dynamic range of protein expression with increasing inducer), and can be used to regulate multiple genes simultaneously (Callura et al., 2010).

Relative to other microbial systems, the use of cyanobacterial riboregulators is just beginning to become more wide-spread and only a few riboswitch designs are in common use for metabolic engineering applications (Connor and Atsumi, 2010). A modified theophylline-dependent synthetic riboswitch was first reported in S. elongatus PCC 7942, allowing a strict regulation of protein production in this cyanobacterium (Nakahira et al., 2013). This effective riboswitch has since been implemented in other cyanobacteria such as Synechocystis PCC 6803, Leptolyngbya BL0902, Nostoc PCC 7120, and Synechocystis sp. strain WHSyn (Synechocystis WHSyn) (Ma et al., 2014; Armshaw et al., 2015; Ohbayashi et al., 2016). Similarly, Taton et al. have also developed NOT gate molecular circuits using transcriptional repressors controlled by theophylline-dependent synthetic riboswitches to downregulate gene expression in five diverse strains of cyanobacteria, including three model organisms, Nostoc PCC 7120, Synechocystis PCC 6803, and S. elongatus PCC 7942, as well as two recent isolates, Leptolyngbya BL0902 and Synechocystis WHSyn (Taton et al., 2017). A native cobalamin-dependent riboswitch has been reported in Synechococcus PCC 7002 (Pérez et al., 2016), although it remains unclear if this genetic 
tool can be implemented in other cyanobacteria that are not cobalamin auxotrophs. More recently, another native riboswitch, a glutamine aptamer, has been described in Synechocystis PCC 6803 (Klähn et al., 2018). However, it exhibited poor ligand affinity compared to the aptamers of other riboswitch classes. These newly-described native regulatory sequences open up the possibility of discovering new cyanobacterial riboswitches still not identified. Future approaches that focus on the combination of in silico structural analysis and in vivo genetic tuning of riboswitches that can be modulated by a wide variety of ligands hold the promise of contributing significantly to metabolic engineering efforts (Berens and Suess, 2015).

\section{Reporter Proteins}

Reporters allow for easy quantitation of gene expression, visualization of subcellular localization, and interaction of proteins with other cellular components (Berla et al., 2013). Fluorescent reporter proteins do not require additional substrates to be detected in vivo and there are an ample range of colors. However, the use of fluorophores can be complicated in cyanobacteria due to the autofluorescence of photosynthetic pigments. The phycobilins and chlorophyll molecules that are major components of the photosynthetic electron transport chain can cause competitive absorbance of excitation light source, reabsorbance of fluorescent or bioluminescent reporter emission, and signal interference (Yokoo et al., 2015; Ruffing et al., 2016). Because of strong chlorophyll autofluorescence, use of red fluorophores is not recommended, but many other reporters including GFPmut3B (a mutant of green fluorescent protein) and EYFP (enhanced yellow fluorescent protein) have been routinely used (Pédelacq et al., 2006; Huang et al., 2010; Yang et al., 2010; Heidorn et al., 2011; Huang and Lindblad, 2013; Landry et al., 2013; Cohen et al., 2014). Despite the frequent use of fluorescent proteins in cyanobacteria and the continual development of improved variants, further improvements to available reporters could greatly increase their range of applications (Rodriguez et al., 2017). Recently developed fluorophores with superior brightness, photostability, and quantum yield have great promise, including mOrange, mTurquiose, mNeonGreen, or Ypet (Chen et al., 2012; Ruffing et al., 2016; Jordan et al., 2017). In addition to fluorescent proteins, luciferase-based bioluminescence assays are routinely used, especially for tracking gene expression patterns throughout the circadian cycle or under different environmental conditions (Fernández-Piñas et al., 2000; Cohen et al., 2015). Luciferase reporters are ideal for monitoring gene expression because of the short half-life of the enzymes, which provides a readout that is close to real-time (Ghim et al., 2010). Alternatively, fluorophores can be modified with protein degradation sequences that greatly reduce their half-life (Wang et al., 2012), increasing their suitability as a transcriptional readout (Noguchi and Golden, 2017).

\section{Modular Vector Systems for Engineering Cyanobacteria}

Vector systems based on the use of standard biological parts (http://parts.igem.org) and assembly schemes (e.g., BioBrick and BglBrick) were originally designed for organisms such as E. coli,
B. subtilis, or yeast in order to increase the modular assembly of many different parts. By contrast, most standard genetic tools and vectors were developed for a specific cyanobacterial strain and generally, these tools have not been designed to be modular. In recent years, a few vector systems have been designed specifically to work in diverse strains and/or to contain a modular organization, which could facilitate standardization and characterization of component parts.

A notable modular vector system was described in 2014, using a range of plasmids designed to be compatible with a broad host-range (Taton et al., 2014). These plasmids included both autonomously replicating plasmids and suicide plasmids for gene knockout and knockin, and were characterized in diverse cyanobacterial strains to ensure their proper functioning. As a part of this work, the authors also created a web server, CYANO-VECTOR, that can assist in the in silico design of plasmids and assembly strategies. Similarly, chromosomal integration vectors carrying standard prefix and suffix sequences suitable for BioBrick-based cloning have been designed for Synechococcus PCC 7002 (Vogel et al., 2017) and S. elongatus PCC 7942 (Kim et al., 2017). In a recent article, a versatile system called CyanoGate based on the Plant Golden Gate MoClo kit and the MoClo kit for the microalgae Chlamydomonas reinhardtii was developed (Vasudevan et al., 2018). Vasudevan et al. demonstrated that the functionality of this system was robust across different two cyanobacterial species, Synechocystis PCC 6803 and S. elongatus UTEX 2973.

\section{Markerless Selection as a Tool to Facilitate Cyanobacterial Engineering}

In cyanobacteria, as in other bacteria, metabolic engineering involving multiple genetic manipulations requires multiple selective markers. To make deletions, genes are normally replaced by antibiotic resistance markers to evaluate what phenotypic effects take place. However, the generation of a strain with numerous deletions is restricted using this method because the availability of resistance markers is limited. Alternatively, a range of markerless selection strategies has been developed to increase the number of modifications that may be performed to modify genetically these organisms.

The first markerless system described in the cyanobacterium $S$. elongatus PCC 7942 relies on a dominant streptomycin-sensitive rps12 mutation (Matsuoka et al., 2001). The method is based on a double selection cassette composed of a kanamycin resistance gene and, as an alternative negative selection marker, a rps12 wild-type copy that confers a dominant streptomycin sensitive phenotype. Streptomycin-resistant, kanamycin-sensitive markerless mutants can be recovered in a second transformation (Takahama et al., 2004). The main drawback of this method is the need to work in a genetic background that contains the appropriate rps12 mutation. Moreover, this strategy requires two, time-consuming transformation events and cloning of two different suicide vectors. Recently, new time-saving alternatives have been developed for markerless gene replacement in cyanobacteria. 
Begemann et al. described a counter-selection method for Synechococcus PCC 7002 based on organic acid toxicity (Begemann et al., 2013). The system was based on the use of the product of the acs $A$ gene, an acetyl-CoA ligase. The loss of AcsA function was used to develop an acrylate counterselection method. Another alternative counter-selection method was developed for Synechocystis PCC 6803, which involves the use of the endogenous nickel inducible promoter to drive an E. coli derived toxin gene known as mazF (Cheah et al., 2013). $\mathrm{MazF}$ is an endoribonuclease that acts as a global inhibitor for the synthesis of cellular proteins, because it cleaves mRNA at the ACA triplet sequence. A different markerless gene deletion system that only requires a single vector has also been described for Synechocystis PCC 6803 and uses a nptI-sacB double selection cassette (Viola et al., 2014). The nptI gene confers resistance to the antibiotic kanamycin, while expression of the $s a c B$ gene is toxic to bacteria grown on sucrose-containing media. Counterselection based on $s a c B$ is not functional in Synechococcus PCC 7002 (Zhang and Song, 2018), possibly because sacB selection is sensitive to salt (Kunst and Rapoport, 1995) required for growth of this marine cyanobacterium. A few markerless gene deletion systems have been shown to work in multiple cyanobacterial strains, for example Kojima et al. developed an efficient method for generating knockouts in Synechocystis PCC 6803 and Synechococcus PCC 7002 (Kojima et al., 2016). This system is based on knocking out the aas gene, an acyl-acyl carrier protein synthetase, and selecting the mutants by their free fatty acid tolerance.

The most recent markerless systems are based on CRISPRbased technology (Figure 2) that does not require any counterselection genes (Behler et al., 2018). In general, all CRISPRCas [clustered regularly interspaced short palindromic repeats (CRISPR)/CRISPR-associated protein (Cas) system] technology relies on the capacity to target a protein (typically a nuclease) to a very precise genomic locus. This specificity is conferred by a single-guide RNA (sgRNA) is programmed to be a complement to the target genomic site, and which assembles with Cas9 or Cas12a (formerly known as Cpf1) into an effector complex (Figure 2A). Depending upon the CRISPR system, the effector complex may contain other RNA sequence: the CRISPR-Cas9 system requires two separate RNA strands, the CRISPR RNA (crRNA) that encodes the guide sequence, and trans-activating crRNA (tracrRNA), while CRISPR-Cas12a requires only a single crRNA (Figure 2A). Most cyanobacterial genomes naturally contain CRISPR-Cas repeat sequences (Cai et al., 2013) useful in defending the cell against foreign genetic material as the Cas nuclease can be directed to cleave sequences that are specific to an exogenous source (e.g., viral DNA).

The specificity of Cas9 and Cas12a targeting has been repurposed to design markerless genome editing systems, and other genetic control elements (Li et al., 2016; Ungerer and Pakrasi, 2016; Wendt et al., 2016; Niu et al., 2018; Ungerer et al., 2018; Xiao et al., 2018). When directed to a DNA target within the cyanobacterial genome, the double-stranded DNA breaks induced by the CRISPR effector are lethal unless they can be repaired in a manner that alters the DNA so that it is no longer recognized by the sgRNA (Figure 2A) (Behler et al., 2018).
Double-stranded DNA breaks engage cyanobacterial DNA repair machinery, which can resolve the damage by error-prone nonhomologous end joining or by homologous recombination if a suitable template is available. To induce an inactivating mutation in a target gene it is often sufficient to express Cas9 or Cas12a along with a gene-specific sgRNA and to rely upon the error in the genetic repair systems to introduce point mutations and frameshifts that will inactivate the gene without the need for a selectable marker. More advanced genome editing (e.g., knockout of specific regions or insertion of new DNA at the target locus) can be accomplished through homologous recombination if a suitable template is also introduced into the cell (see Figure 2B). This markerless methodology has been used to introduce point mutations, knock-out large genomic regions, and "knock-in" genes in a range of cyanobacterial species, including Synechocystis PCC 6803 (Xiao et al., 2018), S. elongatus UTEX 2973 (Ungerer and Pakrasi, 2016; Wendt et al., 2016), S. elongatus 7942 (Li et al., 2016; Ungerer et al., 2018), and Nostoc 7120 (Ungerer and Pakrasi, 2016; Niu et al., 2018).

The CRISPR-Cas system is still under active development and some limitations may need to be overcome before it becomes a methodology that completely replaces more traditional cyanobacterial genome engineering techniques. One limitation is that colonies recovered from CRISPR-mediated transformations can have a low penetrance of the desired genomic alteration. For example, early reports of CRISPR editing in cyanobacteria have shown between 20 and $70 \%$ of recovered strains are the desired mutant (Li et al., 2016; Ungerer and Pakrasi, 2016; Wendt et al., 2016; Xiao et al., 2018). The low efficiency of some transformations requires screening and validation of a higher number of recovered colonies to obtain the correct strain. It may be possible to overcome this limitation by encoding two spacers that target the genomic region rather than one (see spacer depiction in Figure 2B), as shown in recent report in Nostoc PCC 7120 (Niu et al., 2018). The expression of Cas9 also appears to be toxic in a dose-dependent manner in some cyanobacteria, such as $S$. elongatus UTEX 2973 (Wendt et al., 2016), although this toxicity is not apparent in others (Xiao et al., 2018), even in some closely-related species ( $\mathrm{Li}$ et al., 2016). While the mechanism by which Cas9 causes toxicity remains unclear, it is possible to substitute Cas12a for Cas9 to bypass the issue in many species (Ungerer and Pakrasi, 2016; Swarts and Jinek, 2018). Yet, such uncertainties also contribute to the concern that CRISPRmediated techniques can lead to alter off-target genomic sites that could lead to misinterpretation of observed phenotypes (Fu et al., 2013). Strategies to minimize off-target CRISPR-Cas activity have been extensively explored in other organisms, but have not been rigorously evaluated in cyanobacteria. Finally, although genomic modifications introduced by CRISPR are themselves markerless, the sgRNA and nuclease themselves are often introduced on plasmids that require selectable markers (Figure 2B), and following genome editing, it is often desirable to cure the plasmid through some form of counter-selection (Xiao et al., 2018). This can make the process of genome editing by CRISPR-Cas considerably longer than traditional selectionbased approaches. 


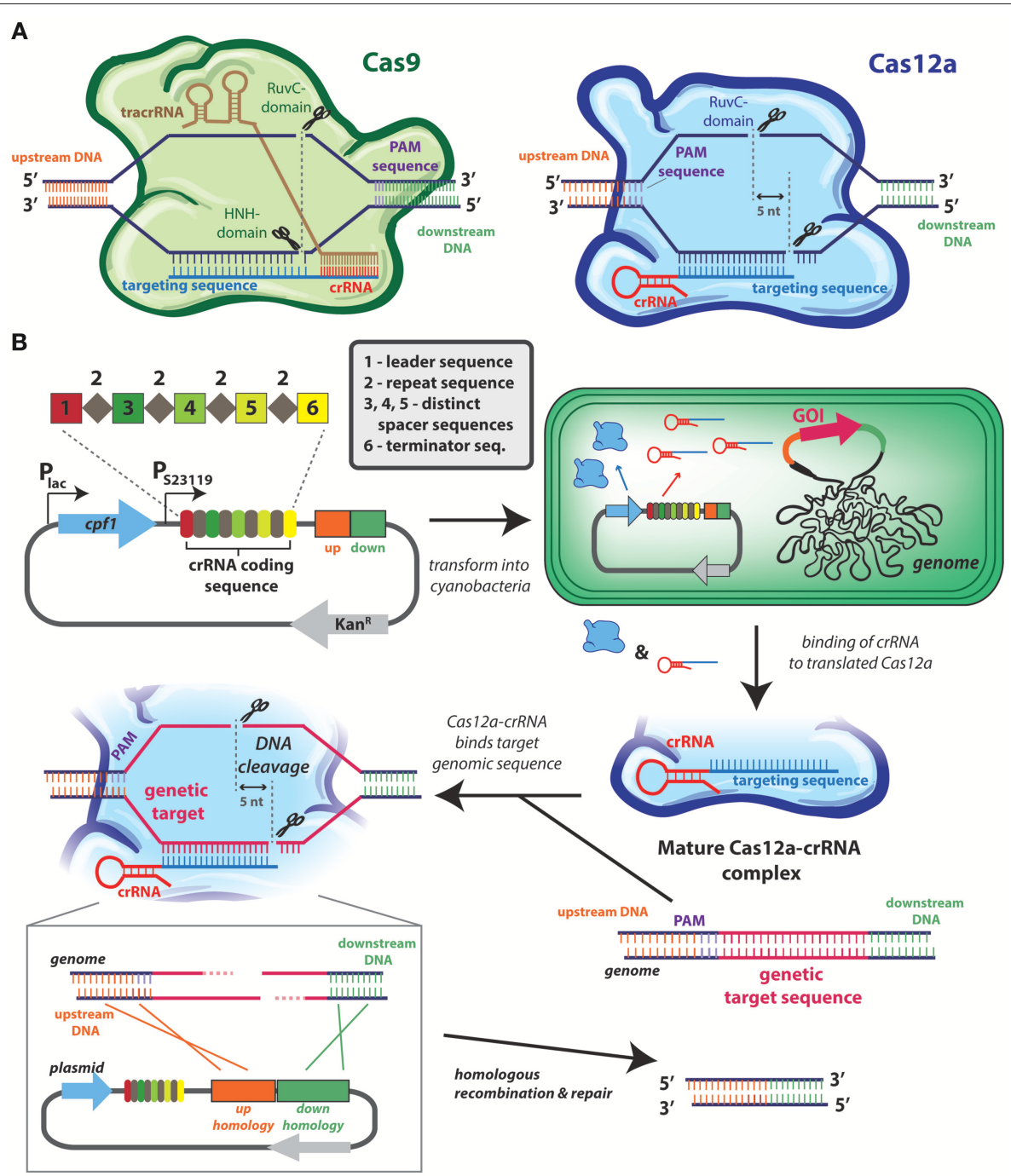

FIGURE 2 | CRISPR-cas technologies used for genome editing of cyanobacterial genome. (A) CRISPR-cas systems used in cyanobacteria: Cas9 (left) and Cas12a (right). In type II systems, Cas9 (green) forms a complex with the crRNA (red) and tracrRNA (brown), whereas in type V systems, Cas12a (blue) forms a complex with its crRNA (red). Both nucleases identify their target sites on the basis of complementarity of the guide sequence (blue) by heteroduplex formation, as well as the presence of a neighboring protospacer-adjacent motif (PAM; purple), although the positioning of the PAM is distinct between systems Cas 9 cleaves the complementary DNA strand using its HNH domain, and a second RuvC domain to cleave the non-complementary strand. Cas12a cleaves target DNA using one RuvC endonuclease domain, and a second endonuclease activity is attributed to a novel nuclease domain. (B) Example application of CRISPR/Cas12a in Synechococcus elongatus PCC 7942. This system is based on the use of a single plasmid that contains: the cas 12a gene under the lac promoter and a crRNA-encoding array (from Francisella novicida) under a constitutive (J23119) promoter. This plasmid allows for inducible, transient expression of cas12a. This crRNA-encoding array contains a leader sequence (1) followed by direct repeat (2) and spacer $(3,4,5)$ sequences, and a terminator (6). In most cyanobacterial CRISPR-Cas reports a single spacer region (e.g., number 3) acts as the genome target spacer, while other spacers are not utilized. Multiple genomic regions can be targeted by encoding other spacers with complementary sequences, or the efficiency of modifying a single genomic loci can be improved by utilizing multiple spacers (Niu et al., 2018). Homology regions designed to promote recombination-mediated repair can also be encoded in the plasmid (represented by orange and green rectangles). Once inside the cell, an active and mature Cas12a-crRNA complex forms and is directed to the target sequence. After the introduction of the 5-nt staggered double-stranded break, the homology regions in the plasmid serve as a repair template to introduce the intended genomic changes. The transconjugants are selected on BG11 agar plates supplemented with kanamycin. The positive colonies are streaked on BG11 agar plates to cure the plasmid and they are assayed to check the loss by the inability to grow on kanamycin-containing media. Depicted system is modeled after the one described by Ungerer and Pakrasi (2016).

CRISPR-based applications reach beyond markerless genome engineering to a wider range of applications. Briefly, nucleasedead variants of Cas9/Cas12a do not generate DNA breaks, but can still be targeted to specific DNA sequences via sgRNA can be fused to other functional domains (e.g., transcriptional activator or repressor domains). This creates hybrid Cas proteins that bind to endogenous sequences, but which can perform other functions, such as regulating gene expression. Recently, these approaches have been used to create synthetic transcription factors able to modulate the expression of essential 
genes and key metabolic pathways in cyanobacteria. For a more comprehensive review of CRISPR-based applications in cyanobacteria, see (Behler et al., 2018).

\section{IMPROVING CARBON CAPTURE AND DIRECTING CARBON FLUX THROUGH RATIONAL ENGINEERING APPROACHES}

Engineering cyanobacterial strains for bioproduction requires that not only heterologous genes and pathways be expressed in new hosts, but also that carbon that is captured by cyanobacteria be efficiently directed toward the desired metabolic products (Stephanopoulos, 2012; Woo, 2017). Thus, it is a prerequisite for metabolic engineers to optimize pathways to increase the total carbon flux toward target product generation, and/or to enhance the pool size of rate-limiting metabolites (Kanno et al., 2017; Carroll et al., 2018). Although the regulation of carbon partitioning in cyanobacteria is not fully understood, it can be diverted under certain conditions like nutrient deprivation and irradiance stress, and therefore stress conditions are routinely used in studies that aim to increase product yield (Woo, 2017). New predictive tools that can assist in channeling fixed carbon to desired metabolic pathways have been in active development. These tools include models for metabolic analysis and flux balance analysis and will be described in greater detail below. Beyond channeling carbon flux, another strategy is to improve the total pool of available carbon, and therefore multiple efforts to improve carbon fixation rates of cyanobacteria have been pursued.

\section{Improving Carbon Fixation Rates}

Many efforts to engineer cyanobacteria for enhanced productivity have emphasized increasing the total rate of carbon fixation, most frequently by improving the activity or efficiency of RuBisCO. Cyanobacteria have highly efficient carbon concentration mechanisms (CCM) relative to other phototrophs such as algae and plants (Price et al., 2011). The cyanobacterial CCM is efficient in part because it uses bicarbonate transporters to actively transport bicarbonate into the cell, which effectively overcomes the slower $\left(10^{4}\right.$-fold $)$ diffusion rates of $\mathrm{CO}_{2}$ in water compared to air (Price et al., 2011). Ultimately, the accumulated bicarbonate is transported across the carboxysome shell, and converted to $\mathrm{CO}_{2}$ in the carboxysome lumen, where RuBisCO is concentrated (Badger et al., 2002; Price et al., 2008). Increasing the efficiency of the cyanobacterial CCM could not only increase the concentration of RuBisCO's substrate, but also reduce the production of energetically-costly photorespiratory byproducts. Recently, extra bicarbonate transporters were expressed in Synechocystis PCC 6803, leading to a 2 -fold enhancement of the growth rate and a higher amount of biomass accumulation (Kamennaya et al., 2015).

Other efforts have focused upon improving carbon fixation rates by balancing the enzymatic activities in the CalvinBenson-Bassham (CBB) cycle to improve its total metabolic flux. Liang and Lindblad have demonstrated that overexpression of any of four separate enzymes in the CBB cycle (RuBisCO,
Sedoheptulose bisphosphatase, fructose bisphosphate aldolase, or transketolase) can improve total carbon fixation rates in Synechocystis (Liang and Lindblad, 2016) (Figure 3). Furthermore, modifying the expression of these CBB enzymes could also enhance the rate of heterologous production of ethanol in Synechocystis PCC 6803 (Liang et al., 2018). Kanno et al. have recently described a novel strategy to improve carbon fixation rates by focusing upon importing a continuous supply of substrate for RuBisCO. By expressing glucose transporters, they successfully used the oxidative pentose phosphate pathway to convert some imported sugars for the synthesis of D-ribulose 1,5 bisphosphate. Using this approach, they demonstrated that an engineered strain was able to produce a targeted biochemical, 2,3-butanediol, under both light and dark conditions (Kanno et al., 2017).

\section{Improving Carbon Fixation Through Sink Engineering}

While it is frequently assumed that the rate limiting metabolic step of carbon fixation is directly related to the slow catalytic activity of RuBisCO, photosynthetic activity can be limited by other metabolic steps. Utilization of the primary products of the CBB cycle can limit step photosynthesis rather than the fixation of carbon dioxide itself. This is concept was first explored in plant models, where the mechanisms underlying triose phosphate utilization (TPU) limitation are more deeply characterized. In plants, there is a physical separation between where primary products of the CBB are generated (source tissues, e.g., leaves) and where much of these products will ultimately be utilized to support metabolic activity and growth (sink tissues; e.g., roots). Efficient operation of photosynthetic metabolism requires the balance of light energy ("source") that can be highly dynamic in the environment with an equivalent capacity to utilize/dissipate this energy using anabolic metabolism or quenching mechanisms ("sinks"). Without absorption of adequate photons (i.e., low "source"), a photosynthetic organism will starve; and without sufficient pathways to process or dissipate absorbed energy (low "sink"), end products of photosynthesis can accumulate leading to feedback inhibition and overreduction of the electron transport chain (ETC) (Gifford et al., 1984; Paul and Foyer, 2001). TPU limitation on photosynthesis can occur either because of experimental manipulation (e.g., exogenously supplied carbohydrates, or chemical inhibition of carbohydrate transport/catabolism), or because the sum of metabolic processes downstream the $\mathrm{CBB}$ are insufficient to remove triose phosphates at an equivalent rate as they are being generated (Sawada et al., 1986; Sharkey et al., 1986; Krapp et al., 1991; Paul and Foyer, 2001; Adams et al., 2013; Demmig-Adams et al., 2014).

While a number of published reports in plants have sought to enhance biomass accumulation by reducing TPU limitation, relatively few studies exist that suggest that cyanobacterial photosynthesis may also be limited by downstream metabolism. Much of our current knowledge on sink limitation in cyanobacteria is indirect, where researchers have found the activity of a heterologous metabolic pathway expressed in a cyanobacterial model leads to increased photosynthetic activity 


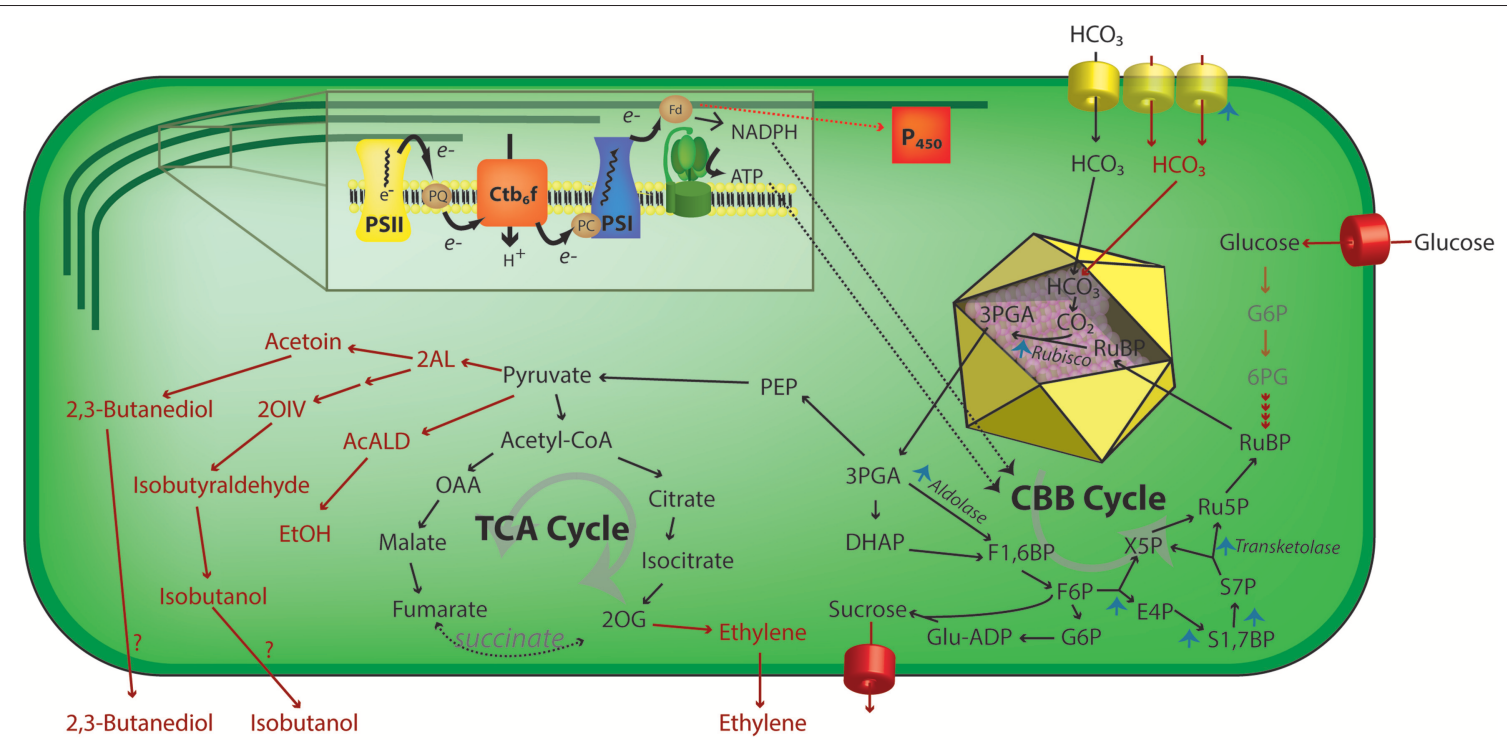

FIGURE 3 | Schematic representation of engineering strategies that increase photosynthetic activity in cyanobacteria. Using ATP and NADPH produced by the light reactions (inset), the $\mathrm{CBB}$ acts to assimilate inorganic carbon via a RuBisCO-mediated reaction in the carboxysome (yellow icosahedron) lumen. Upregulation of multiple steps of the carbon concentration mechanism or CBB cycle enzymes has been shown to increase total carbon uptake rates by cyanobacteria (blue arrowheads). Additionally, the regeneration of RuBisCO substrate, ribulose 1,5-bisphosphate, by feeding external carbohydrates has been used to increase $\mathrm{CO}_{2}$ assimilation rates. Multiple metabolic pathways (red arrows) that have been heterologously expressed in cyanobacteria have also been demonstrated to increase the effective rate of carbon fixation, perhaps by relieving sink inhibition of photosynthesis. 2AL, 2-acetolactate; 2 OIV, 2-ketoisovalerate; 2OG, 2-oxoglutarate; 3PGA, 3-phosphoglycerate; 6PG, 6-phosphoglycerate; AcALD, acetaldehyde; Ctb6f, cytochrome b6f; E4P, erythrose 4-phosphate; EtOH, ethanol; F1,6BP, fructose-1,6-bisphosphate; F6P, fructose-6-phosphate; Fd, ferredoxin; G6P, glucose 6-phosphate; Glu-ADP, Glucose-ADP; OAA, oxalacetate; P450, cytochrome P450; PC, plastocyanin; PEP, phosphoenolpyruvate; PQ, plastoquinone pool; PSI, photosystem I; PSII, photosystem II; Ru5P, ribulose-5-phosphate; S1,7BP, sedoheptulose-1,7-bisphosphate; S7P, sedoheptulose-7-phosphate; TCA, tricarboxylic acid; X5P, xylulose-5-phosphate.

and/or quantum efficiency (Ducat et al., 2012; Oliver et al., 2013). For example, expression of transporters that allow export of a number of bioproducts in cyanobacteria can lead to increases in photosynthetic activity relative to a parental line that lacks the production pathway. Specifically, some degree of enhanced photosynthetic activity has been in diverse cyanobacterial strains engineered to export sucrose (Ducat et al., 2012), isobuteraldehyde (Li et al., 2014), 2,3-butanediol (Oliver et al., 2013), or ethylene (Ungerer et al., 2012) (Figure 3; red text and arrows).

Our group has recently reported a more comprehensive analysis on the photosynthetic effects that occur following activation of a heterologous carbon sink (i.e., sucrose production and secretion) in S. elongatus PCC 7942 (Abramson et al., 2016). In this analysis, we found that photosystem II and photosystem I activities were significantly increased within hours of activating the heterologous sucrose secretion pathway. The quantum efficiency of photosystem II transiently increased following sucrose export and photosystem I activity became less constrained by acceptor-side limitations (i.e., the ability of electron carriers such as ferredoxin to remove the excited electrons generated at the reactive chlorophyll pair of photosystem I; Abramson et al., 2016) and total $\mathrm{CO}_{2}$ fixation rates increased (Ducat et al., 2012). Taken together, these results suggest that overall electron flux through the ETC is enhanced following activation of a heterologous export pathway, suggesting that the endogenous metabolism of S. elongatus PCC 7942 can be insufficient to completely utilize the products of the light reactions under standard laboratory conditions. Similar studies have shown that heterologous electron sinks also have potential to enhance photosynthesis. When a mammalian cytochrome P450, CYP1A1, was expressed in Synechococcus PCC 7002 it was able to utilize reductant from the ETC to catalyze a desired monooxygenation reaction and introduction of this pathway was also associated with an improved photosynthetic efficiency and increased electron flow rate by up to $\sim 30 \%$ (Berepiki et al., 2016).

From the above discussion, it appears that at least slowergrowing strains of cyanobacteria may be limited by their capacity to utilize products of the $\mathrm{CBB}$ cycle. However, it remains unknown if the fastest-growing strains of cyanobacteria (e.g., S. elongatus UTEX 2973 and S. elongatus PCC 11801) will also exhibit similar increases in photosynthetic flux when engineered to export bioproducts. Current evidence suggests that a greater flux of carbon is allocated away from storage products (e.g., glycogen) and instead invested in cell growth and light-harvesting/carbon-fixation machinery in fast-growing cyanobacteria (Mueller et al., 2017; Zhang et al., 2017; Jaiswal et al., 2018). It is possible that strains with naturally high growth rates will experience less photosynthetic limitations due to overaccumulation of $\mathrm{CBB}$ end products, and therefore not exhibit similar enhancements in photosynthesis upon activation of a heterologous metabolic sink. 


\section{PREDICTING AND ENGINEERING CYANOBACTERIAL METABOLISM VIA GENOME-SCALE MODELS}

Genome-scale models (GSMs) are large-scale stoichiometric models that describe metabolic pathways as stoichiometric coefficients and mass balances of participating metabolites, and are simulated using numerical optimization (Kim et al., 2016). The ultimate goal of these metabolic reconstructions is to give a comprehensive explanation of all biochemical conversions taking place within a living cell or organism, including transport and non-enzymatic reactions (Steuer et al., 2012). Due to ease of implementation and relatively high predictive power, modeling approaches have been used as tools to assist metabolic engineering and production strain development (O’Brien et al., 2015). Computational modeling methods based on the use of GSMs complement experimental research and give a powerful tool to rapidly generate and prioritize testable hypotheses that can be used to guide subsequent experimentation (Dreyfuss et al., 2013). On the other hand, GSMs also provide potential mechanistic explanations for the results obtained in the laboratory (O'Brien et al., 2015).

Using the genome sequence of an organism, a draft GSM can be relatively easily compiled (Thiele and Palsson, 2010). Standard procedures have been detailed in the literature, to generate high-quality GSM reconstructions (Feist et al., 2009; Thiele and Palsson, 2010). Moreover, many steps of the reconstruction process have been successfully automated by several software programs (Hamilton and Reed, 2014). This progress has allowed the fast reconstruction of draft GSMs of multiple species (Kim et al., 2016) (Figure 4, Central panel). However, some manual evaluation and curation is required to ensure a high-quality reconstruction (Hamilton and Reed, 2014).

\section{An Overview of Metabolic Models Developed for Cyanobacteria}

A number of GSMs of phototrophic organisms have been published in the last decade, but they are still underrepresented in comparison to heterotrophic microorganisms (Gudmundsson et al., 2017). At the time of this writing, genomes for $>270$ cyanobacterial species have been sequenced (Fujisawa et al., 2017). However, a limited number of cyanobacterial reconstructions have been made available and preliminary models have only been refined for only a handful of species (Table 2). The earliest metabolic reconstructions were based on biochemical data, focusing mainly on central carbon metabolism and photosynthetic pathways (Figure 4, left panel). However, the recent advances in genome sequencing have allowed the generation of metabolic models at the genome scale. A general naming convention used to describe in silico models has been proposed with the form " $i \mathrm{XXxxx";} \mathrm{where} \mathrm{"} i$ " refers to an in silico model, "XX" are the initials of the person who developed the model and "xxx" the number of genes included in the model (Reed et al., 2003). However, many of the published GSMs do not follow this rule. Moreover, most of them lack the universal metabolite and reaction conventions in the network model. This lack of consistency impedes direct information extraction between different models; maintenance and adherence to a universal standard would greatly improve the updating and curation of GSMs. The inconsistent nomenclature is a key bottleneck in the speed of reconstruction of new high quality GSMs (Kumar et al., 2012).

Among cyanobacteria, Synechocystis PCC 6803, is the most extensively studied and well-modeled cyanobacterium, with a total of 12 GSMs (Table 2). Network reconstruction is an iterative process and the most robust models are generally created by gradually expanding and updating a prior draft when new data and tools are available (Gudmundsson et al., 2017). This is the case of GSMs: iSyn811 (Montagud et al., 2011), iSyn731 (Saha et al., 2012), iHK677 (Knoop et al., 2013), and imSyn716 (Gopalakrishnan et al., 2018). While distinct cyanobacterial species have unique characteristics, there are common pathways and core carbon metabolic processes that tend to be described in most GSM models. These common pathways can be schematically decomposed as: photosynthesis (to produce ATP and NADPH and fix inorganic carbon), glycolysis (to produce ATP, NADH, and generate precursor metabolites), the citric acid cycle (to produce other precursor metabolites), oxidative phosphorylation (to produce ATP), the pentose phosphate pathway (to produce reducing equivalents and precursor metabolites), carbohydrate synthesis and triacylglycerol synthesis (to build cell walls and store carbon), and inorganic nitrogen assimilation (to produce proteins, DNA, RNA, chlorophyll, and other secondary metabolites using the relevant pertinent precursor molecules) (Baroukh et al., 2015).

Unlike obligate heterotrophic microorganisms, cyanobacteria can utilize light and inorganic carbon, in addition to organic compounds, for the generation of energy and metabolic precursors. The complex mechanisms of light capture makes it difficult to represent it as a simple biochemical reaction in a metabolic networks (Baroukh et al., 2015). Accurate modeling of phototrophic metabolism requires a new level of detail, including modeling the process of light harvesting and electron transport through a variety of possible pathways. Some advances in this area have been achieved in recent years. Nogales et al. proposed a modeling approach for photosynthetic electron flow pathways in detail in Synechocystis PCC 6803, including many cyclic electron flow and accessory pathways, enabling the study of photosynthetic processes at the system level (Nogales et al., 2012). In an updated representation of the GSM of Synechocystis PCC 6803, the role of photorespiration in cellular growth and the peculiarities of photosynthetic reactions such as light-dependent oxidative stress were integrated (Knoop et al., 2013). Most recently, the development of an approach to incorporate light absorption that factors in the effects of cell shading was achieved in S. elongatus PCC 7942 by modeling light as a metabolite (Broddrick et al., 2016). On the other hand, Qian et al. incorporated a lightdependent PSI/PSII electron transport rate algorithm in a GSM of Synechococcus PCC 7002, which allowed simulations of photoautotrophic growth at different light intensities (Qian et al., 2017). In a new GSM of Synechocystis PCC 6803, an unconstrained photo-respiratory reaction and a mechanism to 


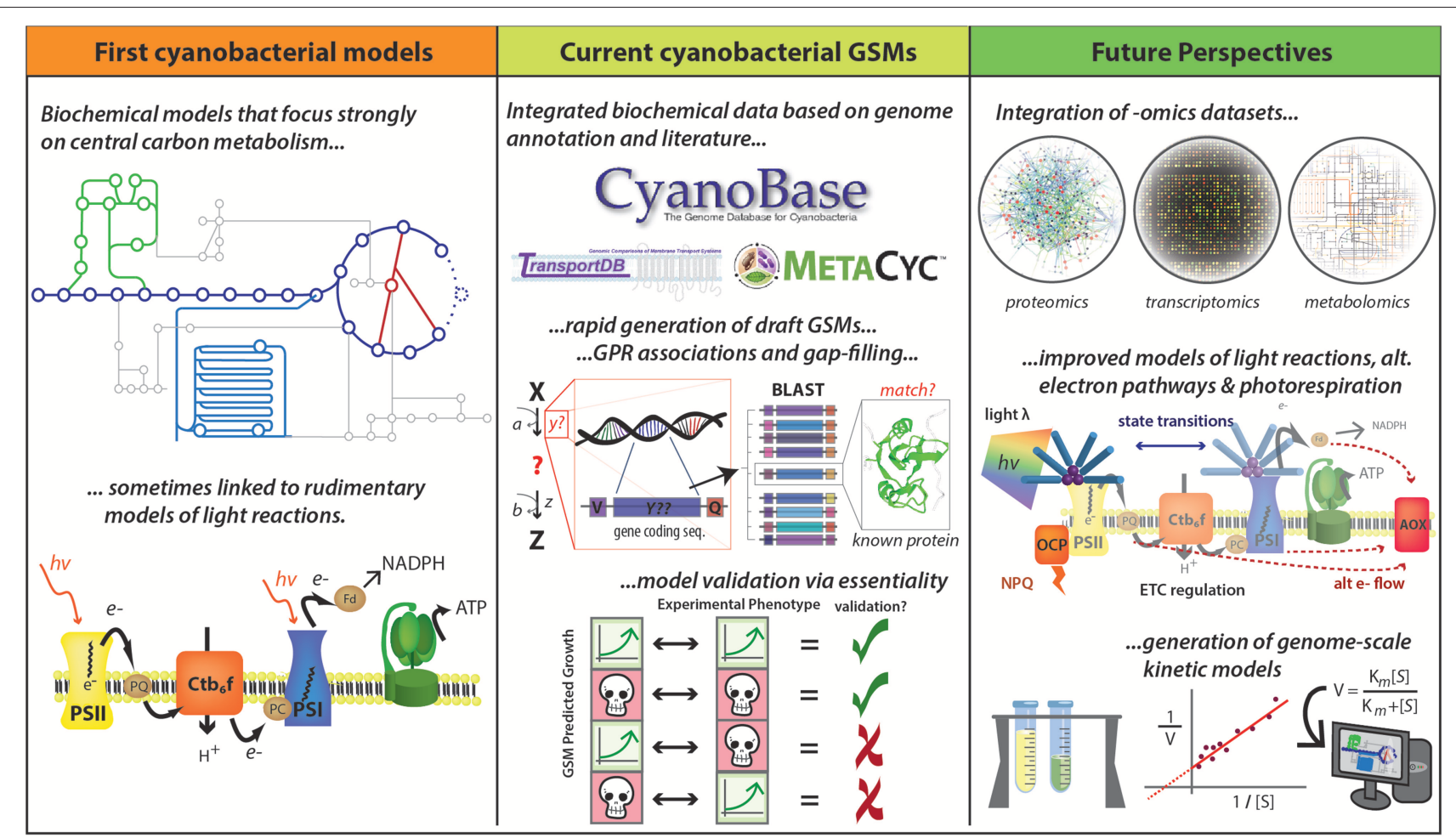

FIGURE 4 | Development of cyanobacterial GSMs. Early cyanobacterial GSMs were developed based on biochemical data and were focused mainly on central carbon metabolism and linked to basic representations of photosynthetic pathways (Cogne et al., 2003; Shastri and Morgan, 2005; Hong and Lee, 2007). Numerous advances have accelerated the reconstruction of GSMs, including improved quantity and quality of data described in public databases (e.g., CyanoBase, TransportDB, KEGG, and MetaCyc) and the development of tools for rapid generation of drafts and for gap-filling. The quality of GSMs has been substantially improved with the inclusion of gene-protein-reaction (GPR) associations, which has been assessed by essential gene prediction based on the availability of experimental data. This methodology allows identify four different kind of predictions: true positives, true negatives, false positives, and false negatives. True positives and true negatives are obtained when both prediction and experimental data indicate a gene is essential and non-essential, respectively (the first and the second case). False positives occur when the model says a gene is essential, but experiments suggest otherwise (the third case). False negatives are generated when a gene is predicted to be non-essential but in reality, it is essential (the fourth case). However, new improvements could further improve GSM predictive ability, including the integration of-omics datasets and the improvement of photosynthesis and photorespiration modeling. A long-term goal would be the development of genome-scale kinetic models, which might be expected to provide more accurate metabolic predictions. Logos for MetaCyc, TransportDB, and CyanoBase are used with permission.

account for changes in energy absorption from light at different wavelengths have been developed (Joshi et al., 2017). To facilitate a better understanding of respiratory and photosynthetic interactions, these authors included features to model known molecular mechanisms of the photosynthetic network around the thylakoid membrane.

A canonical test useful to benchmark and validate the accuracy of GSMs is to examine their capacity to predict essential genes of the metabolic network (Becker and Palsson, 2008) (Figure 4, central panel).Gene essentiality prediction has been successfully used in several bacteria such as E. coli (Suthers et al., 2009; Orth et al., 2011) and Pseudomonas putida (Nogales et al., 2008, 2017). The quality of two recent cyanobacterial GSMs has been assessing using gene essentiality datasets: iJB785 of S. elongatus PCC 7942 (Broddrick et al., 2016) and iSynCJ816 of Synechocystis PCC 6803 (Joshi et al., 2017). In the first case, $78 \%$ of genes were correctly assigned as either essential or non-essential based on data of essentiality in vivo obtained from previous dense-transposon mutagenesis experiments (Rubin et al., 2015). In the second case, the new GSM of Synechocystis PCC 6803 was able to predict gene deletions with $77 \%$ accuracy, based on a qualitative growth comparison of 167 gene-deletion mutants with experimental studies obtained from online databases and a detailed literature search (Joshi et al., 2017).

There are several approaches to improve the accuracy of GSMs. First, the use of data derived from high-throughput growth phenotyping experiments (e.g., knockout mutant strains grown in various media conditions) is very useful for validating and refining metabolic network reconstructions (Gawand et al., 2013). Secondly, integrating of transcriptional regulation has proved to be a vital alternative to build improved models and to investigate the capabilities of reconstructed metabolic networks (Vivek-Ananth and Samal, 2016). Finally, the integration of multi-omics datasets have been used in a number of instances to improve the accuracy of a GSM (Kim and Reed, 2014).

\section{Tools to Improve GSM Quality and Reconstruction}

Manual curation is one the most important steps after the generation of the initial draft of a GSM. The process of manually 
TABLE 2 | GSMs described for cyanobacterial strains.

\begin{tabular}{|c|c|c|c|c|c|c|}
\hline Cyanobacteria & GSM name & Genes & Reactions & Metabolites & $\%$ of annotated genes & References \\
\hline Arthrospira platensis PCC 8005 & $\mathrm{n} / \mathrm{a}$ & $\mathrm{n} / \mathrm{a}$ & 121 & 134 & $\mathrm{n} / \mathrm{a}$ & Cogne et al., 2003 \\
\hline Arthrospira platensis C1 & iAK692 & 692 & 875 & 837 & 11 & Klanchui et al., 2012 \\
\hline $\begin{array}{l}\text { Arthrospira platensis } \\
\text { NIES-39 }\end{array}$ & $\mathrm{n} / \mathrm{a}$ & 620 & 746 & 673 & 24 & Yoshikawa et al., 2015 \\
\hline \multirow[t]{12}{*}{ Synechocystis sp. PCC 6803} & $\mathrm{n} / \mathrm{a}$ & $n / a$ & 70 & 46 & $\mathrm{n} / \mathrm{a}$ & Shastri and Morgan, 2005 \\
\hline & $\mathrm{n} / \mathrm{a}$ & 86 & 56 & 48 & 2.7 & Hong and Lee, 2007 \\
\hline & $\mathrm{n} / \mathrm{a}$ & 633 & 831 & 704 & 20 & Fu, 2009 \\
\hline & $\mathrm{n} / \mathrm{a}$ & 337 & 380 & 291 & 11 & Knoop et al., 2010 \\
\hline & iSyn669 & 669 & 882 & 790 & 21 & Montagud et al., 2010 \\
\hline & iSyn811 & 811 & 956 & 911 & 26 & Montagud et al., 2011 \\
\hline & $\mathrm{n} / \mathrm{a}$ & 393 & 493 & 465 & 12 & Yoshikawa et al., 2011 \\
\hline & iJN678 & 678 & 863 & 795 & 21 & Nogales et al., 2012 \\
\hline & iSyn731 & 731 & 1,156 & 996 & 23 & Saha et al., 2012 \\
\hline & iHK677 & 677 & 759 & 601 & 21 & Knoop et al., 2013 \\
\hline & iSynCJ816 & 816 & 1,045 & 925 & 25.7 & Joshi et al., 2017 \\
\hline & imSyn716 & $\mathrm{n} / \mathrm{a}$ & 729 & 679 & $\mathrm{n} / \mathrm{a}$ & Gopalakrishnan et al., 2018 \\
\hline \multirow[t]{2}{*}{ Cyanothece sp. ATCC 51142} & iCce806 & 806 & 667 & 587 & 16 & Vu et al., 2012 \\
\hline & iCyt773 & 773 & 946 & 811 & 15 & Saha et al., 2012 \\
\hline $\begin{array}{l}\text { Cyanothece sp. } \\
\text { PCC } 7424\end{array}$ & iCyc792 & 792 & 1.242 & 1.107 & 14 & Mueller et al., 2013 \\
\hline $\begin{array}{l}\text { Cyanothece sp. } \\
\text { PCC } 7425\end{array}$ & iCyn731 & 731 & 1.306 & 1.160 & 14 & Mueller et al., 2013 \\
\hline $\begin{array}{l}\text { Cyanothece sp. } \\
\text { PCC } 7822\end{array}$ & iCyj826 & 826 & 1.258 & 1.110 & 14 & Mueller et al., 2013 \\
\hline $\begin{array}{l}\text { Cyanothece sp. } \\
\text { PCC } 8801\end{array}$ & iCyp752 & 752 & 1.172 & 994 & 17 & Mueller et al., 2013 \\
\hline $\begin{array}{l}\text { Cyanothece sp. } \\
\text { PCC } 8802\end{array}$ & iCyh755 & 755 & 1.161 & 973 & 17 & Mueller et al., 2013 \\
\hline Nostoc sp. PCC 7120 & $\mathrm{n} / \mathrm{a}$ & $\mathrm{n} / \mathrm{a}$ & 804 & 777 & $\mathrm{n} / \mathrm{a}$ & Malatinszky et al., 2017 \\
\hline \multirow[t]{2}{*}{ Synechococcus elongatus PCC 7942} & iSyf715 & 715 & 851 & 838 & 26 & Triana et al., 2014 \\
\hline & iJB785 & 785 & 850 & 768 & 29 & Broddrick et al., 2016 \\
\hline Synechococcus elongatus UTEX 2973 & iSyu683 & 687 & 1,178 & 1,028 & 26 & Mueller et al., 2017 \\
\hline \multirow[t]{4}{*}{ Synechococcus sp. PCC 7002} & iSyp611 & 611 & 552 & 542 & 19 & Hamilton and Reed, 2012 \\
\hline & $\mathrm{n} / \mathrm{a}$ & 728 & 742 & 684 & 23 & Hamilton and Reed, 2012 \\
\hline & iSyp708 & 708 & 602 & 581 & 22.2 & Vu et al., 2013 \\
\hline & iSyp821 & 821 & 744 & 777 & 26 & Qian et al., 2017 \\
\hline
\end{tabular}

reconstructing GSMs is complex and requires arduous and time-consuming curation, without which the model quality remains low (Machado et al., 2018). The manual review is required to reflect the true metabolic capabilities of the target organism (Gudmundsson et al., 2017) and relies heavily on experimental, organism-specific information (Thiele and Palsson, 2010). This step involves the examination of the mass and charge balance of individual reactions, gene associations of reactions and reaction directionality (Gudmundsson et al., 2017). This task is typically addressed using the information stored in many public databases such as CyanoBase (Fujisawa et al., 2017), CyanoEXpress (Hernandez-Prieto and Futschik, 2012), KEGG (Kanehisa and Goto, 2000), MetaCyc (Caspi et al., 2006), SEED (Overbeek et al., 2014), BRENDA (Schomburg et al., 2002), and TransportDB (Ren et al., 2004) (Figure 4, central panel). To solve this bottleneck, several tools for rapid automated reconstruction of GSMs are currently available, each offering different degrees of trade-off between automation and human intervention. Some of these tools haven been previously reviewed (Faria et al., 2018; Machado et al., 2018), but only a small number of them have been used in cyanobacteria.

In many cyanobacteria, a high percentage of proteins are annotated as "unknown function" or "hypothetical protein," e.g., in Synechocystis PCC 6803 up to 60\% (Lv et al., 2015). Gene-protein-reaction (GPR) relationships define the association between genes, metabolic enzymes, and the biochemical transformations that they perform (Thomas et al., 2014) (Figure 4, central panel). GPR determines the set of metabolic reactions encoded in the genome and provides a mechanistic link between genotype and phenotype (Machado et al., 2016). The inclusion of GPRs within GSMs is essential to improve the quality of GSMs, with the aim of improving their phenotypic predictions 
(Krishnakumar et al., 2013). New methodologies have been applied to automate the process of adding GPR associations to cyanobacterial GSMs, such as SHARP (Systematic, Homologybased Automated Re-annotation for Prokaryotes). SHARP is a novel PSI-BLAST-based methodology GPR association of the metabolic enzymes involved in prokaryotes, which has been used in cyanobacteria (Krishnakumar et al., 2013). These authors were able to predict 3,781 new GPR associations for the 10 prokaryotes considered, eight of which were cyanobacterial species. These new GPR associations allowed them to annotate gaps in metabolic networks, and to discover several pathways that may be active, thereby providing new directions for metabolic engineering of cyanobacteria.

Another bottleneck during GSM reconstruction is the problem of gap-filling. Metabolic reconstruction via functional genomics (MIRAGE) has been developed specifically to address this problem (Vitkin and Shlomi, 2012), and searches for reactions that are missing from reconstructions purely based on enzyme homology, but where functional genomic data suggests the reactions are present. MIRAGE performance was directly tested by applying it to the reconstruction of a network model for Synechocystis PCC 6803, and was then successfully validated against an existing, manually-curated model for this cyanobacterium. Vitkin and Shlomi compared the reconstructed network model for Synechocystis PCC 6803 generated by MIRAGE with the manually curated models of Knoop et al. (2010) and iSyn811 (Montagud et al., 2011), and found a predictive precision of $70 \%$ in the first case and $37.5 \%$ for iSyn811. MIRAGE was also applied to reconstruct GSMs for 36 sequenced cyanobacteria, including some model cyanobacteria such as S. elongatus PCC 7942 and Synechococcus PCC 7002 (Vitkin and Shlomi, 2012). However, it has been demonstrated that this methodology was not useful to develop a GSM for the cyanobacterium Cyanothece sp. PCC 7424 (Mueller et al., 2013). For example, MIRAGE analysis generated a GSM that contained menaquinone and ubiquinone, compounds shown to not exist within Cyanothece PCC 7424 (Collins and Jones, 1981). Moreover, the biomass composition of this GSM did not contain some metabolites that are known to be important components of this species, including lipids, pigments, and cyanophycin. Automated model development tools are useful when there is enough information in the training set of models that they can extract to develop the new one (Mueller et al., 2013). However, some cyanobacterial metabolites are unique, therefore they cannot be detected using this methodology, and require manual annotation.

\section{Using Flux Balance Analysis for Systems Metabolic Engineering}

The mathematical approach most widely used for studying the characteristics and capabilities of large-scale biochemical networks is flux balance analysis (FBA) (Orth et al., 2010). It depends on an assumption of steady-state growth and mass balance (influx equals efflux) (Orth et al., 2010; Qian et al., 2017). FBA calculates the flow of metabolites through a metabolic network, thereby making it possible to predict the growth rate of an organism or the rate of production of a biotechnologically important metabolite (Orth et al., 2010). In FBA simulations, the biomass function is normally used to simulate cellular growth, because it is composed of all necessary compounds needed to create a new cell including DNA, amino acids, lipids, and polysaccharides (O'Brien et al., 2015). However, when FBA is used to simulate the expected rates of production for a metabolite of interest, instead of maximizing the growth rate, it is necessary to maximize the production of this metabolite by optimizing the output flux of the reaction that produces it (Lewis et al., 2012).

FBA and related constraint-based methods can be used to predict the optimal set of gene knockout and overexpression targets to increase the ability of one organism to produce a chemical of interest. With this aim, FBA has been successfully used in GSMs of cyanobacteria to increase the production of several compounds, as summarized in Table 3. This method is a useful alternative when GSMs have little or no kinetic data available for their metabolic enzymes (Orth et al., 2010). Readers may also find more details about FBA results getting in cyanobacteria in this reference (Gudmundsson et al., 2017) and in the references detailed in Table 3.

\section{Algorithms Used in silico to Improve Cyanobacterial Productivity}

Numerous constraint-based methods of GSMs are available to identify the phenotypic properties of an organism and to validate hypothesis-driven engineering of cellular functions toward specific objectives (Kim et al., 2015). In addition to the increasing refinement of higher-quality GSM reconstructions, other computational algorithms have been developed that have expanded the scope, accuracy, and applications for GSMs. Some of these algorithms have been used in cyanobacteria to predict promising gene deletion targets for increased production of target compounds, such as OptGene, minimization of metabolic adjustment (MOMA), OptKnock, and OptORF ( $\mathrm{Vu}$ et al., 2013; Shabestary and Hudson, 2016). On the other hand, other algorithms have been developed to identify the possible interventions (e.g., up or downregulation of gene expression) that lead to overproduction of a target metabolite (e.g., OptForce) (Shabestary and Hudson, 2016; Lin et al., 2017).

The OptGene algorithm is based on the random implementation of reaction knockouts with the aim to create optimal knockout sets. This algorithm provides the advantage of a high computational speed, enabling solutions to be efficiently reached even for problems of larger size. Additionally, OptGene can optimize for non-linear objective functions, such as the productivity of one specific compound (Patil et al., 2005). This algorithm was recently used to identify knockouts in Synechocystis PCC 6803 that improve production of fermentation, fatty-acid, and terpene-derived biofuels (Shabestary and Hudson, 2016). The same authors also used MOMA, an algorithm that predicts the optimal flux distribution of altered metabolism that would require the smallest change from that of wild-type metabolism (Segrè et al., 2002). However, a primary drawback of the MOMA algorithm is that it does not assume optimality of growth or any other metabolic functions 
TABLE 3 | Applications of FBA to maximize the production of different compounds in cyanobacteria.

\begin{tabular}{|c|c|c|c|}
\hline & Compound & Organism & Reference \\
\hline \multirow[t]{7}{*}{ Alcohols } & 1-propanol & Synechocystis sp. PCC 6803 & Yoshikawa et al., 2011 \\
\hline & Butanol & Synechocystis sp. PCC 6803 & Kämäräinen et al., 2012 \\
\hline & Several alcohols & Synechococcus sp. PCC 7002 & Vu et al., 2013 \\
\hline & $\begin{array}{l}\text { Ethanol, isobutanol, 3-methyl-1-butanol, } \\
\text { 2-methyl-1-butanol and propanol }\end{array}$ & Synechocystis sp. PCC 6803 & Mohammadi et al., 2016 \\
\hline & Butanol, ethanol & Synechococcus sp. PCC 7002 & Hendry et al., 2016 \\
\hline & 1,3-propanediol, glycerol & Synechococcus elongatus PCC 7942 & Hirokawa et al., 2017 \\
\hline & Ethanol & Synechocystis sp. PCC 6803 & Yoshikawa et al., 2017 \\
\hline \multirow[t]{4}{*}{ Bulk chemicals } & Isoprene & Synechocystis sp. PCC 6803 & Saha et al., 2012 \\
\hline & Ethylene & Synechocystis sp. PCC 6803 & Zavrel et al., 2016 \\
\hline & Succinic acid & Synechocystis sp. PCC 6803 & Shirai et al., 2016 \\
\hline & Terpenes & Synechocystis sp. PCC 6803 & Englund et al., 2018 \\
\hline \multirow[t]{3}{*}{ Gases } & Hydrogen & Synechocystis sp. PCC 6803 & Montagud et al., 2010 \\
\hline & Hydrogen & Cyanothece sp. ATCC 51142 & Saha et al., 2012 \\
\hline & Methane & Synechococcus sp. PCC 7002 & Comer et al., 2017 \\
\hline
\end{tabular}

(Raman and Chandra, 2009). Despite of these issues, it has been successfully applied in E. coli to predict the metabolic phenotype of gene knockouts and to find solutions not detected with FBA (Segrè et al., 2002). Shabestary et al. used MOMA to find knockout strategies that could increase biofuel productivity in Synechocystis PCC 6803 (Shabestary and Hudson, 2016). MOMA has been used in Synechococcus PCC 7002 to identify knockout mutants to improve chemical production under photoautotrophic and/or dark anoxic conditions ( $\mathrm{Vu}$ et al., 2013). Shabestary et al. used a third algorithm to find a set of knockouts that led to coupling between biofuel and growth (Shabestary and Hudson, 2016). OptKnock is a powerful algorithm that identifies and subsequently removes metabolic reactions that are capable of coupling cellular growth with chemical production (Burgard et al., 2003). OptORF is an algorithm very similar to OptKnock, but it identifies gene deletions (instead of reaction deletions) and regulatory changes needed to couple growth and chemical production (Kim and Reed, 2010). Nevertheless, the applicability of this algorithm relies heavily on the availability of integrated metabolic and regulatory models, which is not always possible, especially for cyanobacteria (Maia et al., 2016). It was used in Synechococcus PCC 7002 to predict metabolic engineering strategies that improve production of both native and non-native chemicals (Vu et al., 2013).

OptForce is an algorithm that identifies all possible engineering interventions by classifying reactions in the metabolic model depending upon whether their flux values must increase, decrease or become equal to zero to meet a pre-specified overproduction target (Ranganathan et al., 2010). This algorithm has been recently extended to include kinetic descriptions for some of the reaction steps (Chowdhury et al., 2014). The new algorithm, k-OptForce, can only be directly applied when kinetic models of cellular pathways are available and unfortunately detailed kinetic models are extremely limited in cyanobacteria (Steuer et al., 2012). OptForce algorithm was applied in
Synechocystis PCC 6803 to find a set of interventions to couple growth to 1-octanol and limonene production (Shabestary and Hudson, 2016). In the first case, OptForce predicted several reactions in alternative electron flow and required the upregulation of the ferredoxin:NADPH oxidoreductase reaction. In the case of limonene production-growth couple, a significant number of reaction knockouts were required, including some components of the cyclic electron flow and alternative electron flow. In a recent report, Lin et al. used this algorithm in Synechocystis PCC 6803 to enhance isoprenoid production (Lin et al., 2017). OptForce predicted several interventions: the up-regulation of two pentose phosphate pathway genes, ribose 5phosphate isomerase and ribulose 5-phosphate 3-epimerase, and the overexpression of a geranyl diphosphate synthase involved in the limonene biosynthetic pathway. The optimized strain with these modifications demonstrated a 2.3 -fold improvement in productivity.

\section{CONCLUSIONS AND PERSPECTIVES}

Cyanobacteria possess desirable characteristics as a chassis for biotechnological production and have demonstrated capacities to produce high-value bioproducts. However, tools from heterotrophs are not always transferrable to these microorganisms, leading to the delay in their advancement as industrial hosts. Accelerating the development of sophisticated genomic tools is likely to greatly assist in making scaled cyanobacterial bioproduction a commercially-viable endeavor. Many recent efforts have been focused on the characterization of new parts and development of libraries and standardized modular parts in cyanobacteria. However, characterization of such parts could likely be significantly accelerated by the development of robust and modular expression libraries, like those that have been established in E. coli and yeast. Moreover, the development of well-defined genetic libraries such as 
genomic, expression, and knockout libraries might facilitate a better understanding of complex phenotypes in cyanobacteria (Ramey et al., 2015). On the other hand, the use of markerless modification systems as well as CRISPR/Cas based technologies could expand and improve the efficiency of genome editing in cyanobacteria. The iterative modification of the genome would allow the assembly of long and complex metabolic pathways, a capacity that is essential for many of the more advanced and ambitious metabolic engineering projects (Ungerer and Pakrasi, 2016; Behler et al., 2018). The development and/or optimization of high-throughput approaches that allow the introduction of several genetic modifications in a single step are needed (Ramey et al., 2015). In E. coli, techniques such as Multiplex Automated Genome Engineering (MAGE) (Wang et al., 2009) and trackable multiplex recombineering (Warner et al., 2010) have been successfully used to perform multiple genome engineering modifications in tandem. While some technical challenges would be complicate adoption of such techniques in cyanobacteria, it is notable that MAGE has been successfully applied in combination with CRISPR/Cas technology to engineer polyploid hosts, such as industrial yeast (Lian et al., 2018), and other organisms with more complex genomes, including plants (Sakuma et al., 2014; Hashimoto et al., 2018). The establishment of these approaches in higher organisms, together with the recent development of CRISPR/Cas systems in cyanobacteria, open up the possibility of the development of high-throughput genome engineering of cyanobacterial strains.

The increasing availability of GSMs for different cyanobacterial species have allowed for reiterative designbuild-test cycles whereby the predictions from in silico models can be validated and improved from experimental outcomes in vivo. Recent efforts have focused on the establishment of GPRs to improve the quality of GSMs, and thus, their phenotypic predictions. However, there are still some challenges that need to be addressed to ensure the accuracy of GSM predictions. Improving the scope and accuracy of existing GSMs will likely involve the integration of -omic datasets in to improve in silico representation of metabolism and identify additional biological unknowns (Figure 4, right panel). In addition, it is necessary to improve the modeling of photosynthesis, photorespiration, and photodamage (Figure 4, right panel). The correct modeling of photosynthesis and respiratory electron transfer processes might provide some insight into their physiological role in cyanobacteria. Another critical element that is currently absent in GSMs is the incorporation of regulatory pathways that can dynamically alter enzymatic activity and pathway flux (Figure 4, right panel), although in many cases information about regulatory functions is poorly understood in cyanobacteria.

\section{REFERENCES}

Abernathy, M. H., Yu, J., Ma, F., Liberton, M., Ungerer, J., Hollinshead, W. D., et al. (2017). Deciphering cyanobacterial phenotypes for fast photoautotrophic growth via isotopically nonstationary metabolic flux analysis. Biotechnol. Biofuels 10:273. doi: 10.1186/s13068-017-0958-y

Abramson, B. W., Kachel, B., Kramer, D. M., and Ducat, D. C. (2016). Increased photochemical efficiency in cyanobacteria via an engineered sucrose sink. Plant Cell Physiol. 57, 2451-2460. doi: 10.1093/pcp/pcw169
Although existing GSMs have been successfully applied to inform genetic engineering, it is clear that future efforts are being redirected to genome-scale kinetic models, which have stillgreater promise. These new models overcome many of GSM shortcomings, such as the lack of representation of metabolite concentrations and enzymatic regulation, which are necessary for a complete physiologically relevant model. Moreover, they enable dynamic analysis of biological systems for enhanced in silico hypothesis generation (Srinivasan et al., 2015).

Most efforts in the genetic and metabolic engineering of cyanobacteria for the production of compounds with biotechnological applications have been focused on increasing product yield under laboratory conditions. A limited number of studies has been performed with the aim to optimize cyanobacterial cells to be more compatible with downstream scaled cultivation and processing (Singh et al., 2016; Johnson et al., 2017). The successful utilization of cyanobacterial species for industrial production depends on the development of accurate large-scale cultivation systems. However, most academic researchers lack access to large-scale production systems that are necessary to evaluate the potential of engineered strains under more realistic environmental conditions (Schoepp et al., 2014) and such information is important for the recursive designbuild-test process for strain engineering. More communication between academic researchers and industrial partners could assist in the development of accurate equipment to mimic outdoor production conditions at the laboratory scale (as developed in Lucker et al., 2014). In addition to improving product yields, future research should also be addressed to the development of efficient and cost-effective photosynthetic bioreactors (Lau et al., 2015), as well as technologies to harvest the end products (Knoot et al., 2018) to minimize operation costs. Moreover, the high requirements of water and nutrients are other major challenges for economic profitability of large-scale cyanobacterial cultures (Pathak et al., 2018). Further investigation of the capacity of industrial strains of cyanobacteria to grow and remediate wastewater streams might help to alleviate some of these scalability concerns.

\section{AUTHOR CONTRIBUTIONS}

MS-M, AS, and DD contributed to the writing and editing of the manuscript and generation of figures and tables.

\section{FUNDING}

This work was supported by the Department of Energy (Grant: DE-FG02-91ER20021).

Acién, F. G., Molina, E., Reis, A., Torzillo, G., Zittelli, G. C., Sepúlveda, C., et al. (2017). "Photobioreactors for the production of microalgae," in Microalgae-Based Biofuels and Bioproducts, eds C. GonzalezFernandez and R. Muñoz (Kidlington: Woodhead Publishing), $1-44$.

Adams, W. W. III., Muller, O., Cohu, C. M., and Demmig-Adams, B. (2013). May photoinhibition be a consequence, rather than a cause, of limited plant productivity? Photosyn. Res. 117, 31-44. doi: 10.1007/s11120-0139849-7 
Albers, S. C., Gallegos, V. A., and Peebles, C. A. (2015). Engineering of genetic control tools in Synechocystis sp. PCC 6803 using rational design techniques. J. Biotechnol. 216, 36-46. doi: 10.1016/j.jbiotec.2015.09.042

Angermayr, S. A., Gorchs Rovira, A., and Hellingwerf, K. J. (2015). Metabolic engineering of cyanobacteria for the synthesis of commodity products. Trends Biotechnol. 33, 352-361. doi: 10.1016/j.tibtech.2015.03.009

Armshaw, P., Carey, D., Sheahan, C., and Pembroke, J. T. (2015). Utilising the native plasmid, pCA2.4, from the cyanobacterium Synechocystis sp. strain PCC6803 as a cloning site for enhanced product production. Biotechnol. Biofuels 8:201. doi: 10.1186/s13068-015-0385-x

Badger, M. R., Hanson, D., and Price, G. D. (2002). Evolution and diversity of $\mathrm{CO}_{2}$ concentrating mechanisms in cyanobacteria. Funct. Plant Biol. 29, 161-173. doi: 10.1071/PP01213

Baroukh, C., Muñoz-Tamayo, R., Steyer, J. P., and Bernard, O. (2015). A state of the art of metabolic networks of unicellular microalgae and cyanobacteria for biofuel production. Metab. Eng. 30, 49-60. doi: 10.1016/j.ymben.2015.03.019

Becker, S. A., and Palsson, B. O. (2008). Three factors underlying incorrect in silico predictions of essential metabolic genes. BMC Syst. Biol. 2:14. doi: 10.1186/1752-0509-2-14

Begemann, M. B., Zess, E. K., Walters, E. M., Schmitt, E. F., Markley, A. L., and Pfleger, B. F. (2013). An organic acid based counter selection system for cyanobacteria. PLoS ONE 8:e76594. doi: 10.1371/journal.pone.0076594

Behler, J., Vijay, D., Hess, W. R., and Akhtar, M. K. (2018). CRISPR-based technologies for metabolic engineering in cyanobacteria. Trends Biotechnol. 36, 996-1010. doi: 10.1016/j.tibtech.2018.05.011

Berens, C., and Suess, B. (2015). Riboswitch engineering - making the allimportant second and third steps. Curr. Opin. Biotechnol. 31, 10-15. doi: 10.1016/j.copbio.2014.07.014

Berepiki, A., Hitchcock, A., Moore, C. M., and Bibby, T. S. (2016). Tapping the unused potential of photosynthesis with a heterologous electron sink. ACS Synth. Biol. 5, 1369-1375. doi: 10.1021/acssynbio.6b00100

Berla, B. M., Saha, R., Immethun, C. M., Maranas, C. D., Moon, T. S., and Pakrasi, H. B. (2013). Synthetic biology of cyanobacteria: unique challenges and opportunities. Front. Microbiol. 4:246. doi: 10.3389/fmicb.2013.00246

Broddrick, J. T., Rubin, B. E., Welkie, D. G., Du, N., Mih, N., Diamond, S., et al. (2016). Unique attributes of cyanobacterial metabolism revealed by improved genome-scale metabolic modeling and essential gene analysis. Proc. Natl. Acad. Sci. U.S.A. 113, E8344-E8353. doi: 10.1073/pnas.1613446113

Burgard, A. P., Pharkya, P., and Maranas, C. D. (2003). Optknock: a bilevel programming framework for identifying gene knockout strategies for microbial strain optimization. Biotechnol. Bioeng. 84, 647-657. doi: 10.1002/bit.10803

Cai, F., Axen, S. D., and Kerfeld, C. A. (2013). Evidence for the widespread distribution of CRISPR-Cas system in the Phylum Cyanobacteria. RNA Biol. 10, 687-693. doi: 10.4161/rna.24571

Cai, Y., and Wolk, C. P. (1997). Anabaena sp. strain PCC 7120 responds to nitrogen deprivation with a cascade-like sequence of transcriptional activations. J. Bacteriol. 179, 267-271. doi: 10.1128/jb.179.1.267-271.1997

Callahan, S. M., and Buikema, W. J. (2001). The role of HetN in maintenance of the heterocyst pattern in Anabaena sp. PCC 7120. Mol. Microbiol. 40, 941-950. doi: 10.1046/j.1365-2958.2001.02437.x

Callura, J. M., Dwyer, D. J., Isaacs, F. J., Cantor, C. R., and Collins, J. J. (2010). Tracking, tuning, and terminating microbial physiology using synthetic riboregulators. Proc. Natl. Acad. Sci. U.S.A. 107, 15898-15903. doi: 10.1073/pnas.1009747107

Camsund, D., and Lindblad, P. (2014). Engineered transcriptional systems for cyanobacterial biotechnology. Front. Bioeng. Biotechnol. 2:40. doi: 10.3389/fbioe.2014.00040

Cao, Y. Q., Li, Q., Xia, P. F., Wei, L. J., Guo, N., Li, J. W., et al. (2017). AraBAD based toolkit for gene expression and metabolic robustness improvement in Synechococcus elongatus. Sci. Rep. 7:18059. doi: 10.1038/s41598-017-17035-4

Carroll, A. L., Case, A. E., Zhang, A., and Atsumi, S. (2018). Metabolic engineering tools in model cyanobacteria. Metab. Eng. 50, 47-56. doi: 10.1016/j.ymben.2018.03.014

Caspi, R., Foerster, H., Fulcher, C. A., Hopkinson, R., Ingraham, J., Kaipa, P., et al. (2006). MetaCyc: a multiorganism database of metabolic pathways and enzymes. Nucleic Acids Res. 34(Database issue), D511-D516. doi: $10.1093 /$ nar/gkj128
Cassier-Chauvat, C., Veaudor, T., and Chauvat, F. (2016). Comparative genomics of DNA recombination and repair in cyanobacteria: biotechnological implications. Front. Microbiol. 7:1809. doi: 10.3389/fmicb.2016.01809

Cerniglia, C. E., Gibson, D. T., and Baalen, C. (1979). Algal oxidation of aromatic hydrocarbons: formation of 1-naphthol from naphthalene by Agmenellum quadruplicatum, strain PR-6. Biochem. Biophys. Res. Commun. 88, 50-58. doi: 10.1016/0006-291X(79)91695-4

Cerniglia, C. E., Gibson, D. T., and Van Baalen, C. (1980). Oxidation of naphthalene by cyanobacteria and microalgae. J. General Microbiol. 116, 495-500. doi: 10.1099/00221287-116-2-495

Cheah, Y. E., Albers, S. C., and Peebles, C. A. (2013). A novel counter-selection method for markerless genetic modification in Synechocystis sp. PCC 6803. Biotechnol. Prog. 29, 23-30. doi: 10.1002/btpr.1661

Chen, A. H., Afonso, B., Silver, P. A., and Savage, D. F. (2012). Spatial and temporal organization of chromosome duplication and segregation in the cyanobacterium Synechococcus elongatus PCC 7942. PLoS ONE 7:e47837. doi: 10.1371/journal.pone.0047837

Cheng, A. A., and Lu, T. K. (2012). Synthetic biology: an emerging engineering discipline. Ann. Rev. Biomed. Eng. 14, 155-178. doi: 10.1146/annurev-bioeng-071811-150118

Chisti, Y. (2013). Constraints to commercialization of algal fuels. J. Biotechnol. 167, 201-214. doi: 10.1016/j.jbiotec.2013.07.020

Chowdhury, A., Zomorrodi, A. R., and Maranas, C. D. (2014). k-OptForce: integrating kinetics with flux balance analysis for strain design. PLoS Comput. Biol. 10:e1003487. doi: 10.1371/journal.pcbi.1003487

Chungjatupornchai, W., and Fa-Aroonsawat, S. (2014). The rrnA promoter as a tool for the improved expression of heterologous genes in cyanobacteria. Microbiol. Res. 169, 361-368. doi: 10.1016/j.micres.2013.09.010

Cogne, G., Gros, J. B., and Dussap, C. G. (2003). Identification of a metabolic network structure representative of Arthrospira (Spirulina) platensis metabolism. Biotechnol. Bioeng. 84, 667-676. doi: 10.1002/bit.10808

Cohen, S. E., Erb, M. L., Pogliano, J., and Golden, S. S. (2015). Best practices for fluorescence microscopy of the cyanobacterial circadian clock. Meth. Enzymol. 551, 211-221. doi: 10.1016/bs.mie.2014.10.014

Cohen, S. E., Erb, M. L., Selimkhanov, J., Dong, G., Hasty, J., Pogliano, J., et al. (2014). Dynamic localization of the cyanobacterial circadian clock proteins. Curr. Biol. 24, 1836-1844. doi: 10.1016/j.cub.2014.07.036

Collins, M. D., and Jones, D. (1981). Distribution of isoprenoid quinone structural types in bacteria and their taxonomic implication. Microbiol. Rev. 45, 316-354.

Comer, A. D., Long, M. R., Reed, J. L., and Pfleger, B. F. (2017). Flux balance analysis indicates that methane is the lowest cost feedstock for microbial cell factories. Metabol. Eng. Commun. 5, 26-33. doi: 10.1016/j.meteno.2017.07.002

Connor, M. R., and Atsumi, S. (2010). Synthetic biology guides biofuel production. J. Biomed. Biotechnol. 2010:541698. doi: 10.1155/2010/541698

Decoene, T., De Paepe, B., Maertens, J., Coussement, P., Peters, G., De Maeseneire, S. L., et al. (2018). Standardization in synthetic biology: an engineering discipline coming of age. Crit. Rev. Biotechnol. 38, 647-656. doi: 10.1080/07388551.2017.1380600

Demmig-Adams, B., Garab, G., Adams Iii, W., and Govindjee (2014). NonPhotochemical Quenching and Energy Dissipation in Plants, Algae and Cyanobacteria. Dordrecht: Springer.

Dismukes, G. C., Carrieri, D., Bennette, N., Ananyev, G. M., and Posewitz, M. C. (2008). Aquatic phototrophs: efficient alternatives to land-based crops for biofuels. Curr. Opin. Biotechnol. 19, 235-240. doi: 10.1016/j.copbio.2008.05.007

Dittmann, E., Gugger, M., Sivonen, K., and Fewer, D. P. (2015). Natural product biosynthetic diversity and comparative genomics of the cyanobacteria. Trends Microbiol. 23, 642-652. doi: 10.1016/j.tim.2015.07.008

Ditty, J. L., Williams, S. B., and Golden, S. S. (2003). A cyanobacterial circadian timing mechanism. Аnnu. Rev. Genet. 37, 513-543. doi: 10.1146/annurev.genet.37.110801.142716

Dreyfuss, J. M., Zucker, J. D., Hood, H. M., Ocasio, L. R., Sachs, M. S., and Galagan, J. E. (2013). Reconstruction and validation of a genome-scale metabolic model for the filamentous fungus Neurospora crassa using FARM. PLoS Comput. Biol. 9:e1003126. doi: 10.1371/journal.pcbi.1003126

Ducat, D. C., Avelar-Rivas, J. A., Way, J. C., and Silver, P. A. (2012). Rerouting carbon flux to enhance photosynthetic productivity. Appl. Environ. Microbiol. 78, 2660-2668. doi: 10.1128/AEM.07901-11 
Ellis, B. E. (1977). Degradation of phenolic compounds by fresh-water algae. Plant Sci. Lett. 8, 213-216. doi: 10.1016/0304-4211(77)90183-3

Englund, E., Liang, F., and Lindberg, P. (2016). Evaluation of promoters and ribosome binding sites for biotechnological applications in the unicellular cyanobacterium Synechocystis sp. PCC 6803. Sci. Rep. 6:36640. doi: $10.1038 /$ srep 36640

Englund, E., Shabestary, K., Hudson, E. P., and Lindberg, P. (2018). Systematic overexpression study to find target enzymes enhancing production of terpenes in Synechocystis PCC 6803, using isoprene as a model compound. Metab. Eng. 49, 164-177. doi: 10.1016/j.ymben.2018.07.004

Faria, J. P., Rocha, M., Rocha, I., and Henry, C. S. (2018). Methods for automated genome-scale metabolic model reconstruction. Biochem. Soc. Trans. 46, 931-936. doi: 10.1042/BST20170246

Feist, A. M., Herrgård, M. J., Thiele, I., Reed, J. L., and Palsson BØFeist, B. O. (2009). Reconstruction of biochemical networks in microorganisms. Nat. Rev. Microbiol. 7, 129-143. doi: 10.1038/nrmicro1949

Fernández-Piñas, F., Leganés, F., and Wolk, C. P. (2000). Bacterial lux genes as reporters in cyanobacteria. Method Enzymol. 305, 513-527. doi: 10.1016/S0076-6879(00)05510-5

Ferreira, E. A., Pacheco, C. C., Pinto, F., Pereira, J., Lamosa, P., Oliveira, P., et al. (2018). Expanding the toolbox for Synechocystis sp. PCC 6803: validation of replicative vectors and characterization of a novel set of promoters. Synthet. Biol. 3:ysy014. doi: 10.1093/synbio/ysy014

$\mathrm{Fu}$, P. (2009). Genome-scale modeling of Synechocystis sp. PCC 6803 and prediction of pathway insertion. J. Chem. Technol. Biotechnol. 84, 473-483. doi: $10.1002 /$ jctb. 2065

Fu, P. (2013). Grand challenges in synthetic biology to be accomplished. Front. Bioeng. Biotechnol. 1:2. doi: 10.3389/fbioe.2013.00002

Fu, Y., Foden, J. A., Khayter, C., Maeder, M. L., Reyon, D., Joung, J. K., et al. (2013). High-frequency off-target mutagenesis induced by CRISPRCas nucleases in human cells. Nat. Biotechnol. 31, 822-826. doi: 10.1038/nb t.2623

Fujisawa, T., Narikawa, R., Maeda, S. I., Watanabe, S., Kanesaki, Y., Kobayashi, K., et al. (2017). CyanoBase: a large-scale update on its 20th anniversary. Nucleic Acids Res. 45, D551-D554. doi: 10.1093/nar/gkw1131

Gawand, P., Yang, L., Cluett, W. R., and Mahadevan, R. (2013). Metabolic model refinement using phenotypic microarray data. Methods Mol. Biol. 985, 47-59. doi: 10.1007/978-1-62703-299-5_3

Geerts, D., Bovy, A., de Vrieze, G., Borrias, M., and Weisbeek, P. (1995). Inducible expression of heterologous genes targeted to a chromosomal platform in the cyanobacterium Synechococcus sp. PCC 7942. Microbiology 141(Pt 4), 831-841.

Ghim, C. M., Lee, S. K., Takayama, S., and Mitchell, R. J. (2010). The art of reporter proteins in science: past, present and future applications. BMB Rep. 43, 451-460. doi: 10.5483/BMBRep.2010.43.7.451

Gifford, R. M., Thorne, J. H., Hitz, W. D., and Giaquinta, R. T. (1984). Crop productivity and photoassimilate partitioning. Science 225, 801-808. doi: $10.1126 /$ science.225.4664.801

Gopalakrishnan, S., Pakrasi, H. B., and Maranas, C. D. (2018). Elucidation of photoautotrophic carbon flux topology in Synechocystis PCC 6803 using genome-scale carbon mapping models. Metab. Eng. 47, 190-199. doi: 10.1016/j.ymben.2018.03.008

Gordon, G. C., Korosh, T. C., Cameron, J. C., Markley, A. L., Begemann, M. B., and Pfleger, B. F. (2016). CRISPR interference as a titratable, trans-acting regulatory tool for metabolic engineering in the cyanobacterium Synechococcus sp. strain PCC 7002. Metab. Eng. 38, 170-179. doi: 10.1016/j.ymben.2016.07.007

Griese, M., Lange, C., and Soppa, J. (2011). Ploidy in cyanobacteria. FEMS Microbiol. Lett. 323, 124-131. doi: 10.1111/j.1574-6968.2011.02368.x

Grigorieva, G., and Shestakov, S. (1982). Transformation in the cyanobacterium Synechocystis sp. 6803. FEMS Microbiol. Lett. 13, 367-370. doi: 10.1111/j.1574-6968.1982.tb08289.x

Gudmundsson, S., Agudo, L., and Nogales, J. (2017). "Applications of genomescale metabolic models of microalgae and cyanobacteria in biotechnology," in Microalgae-Based Biofuels and Bioproducts, eds C. Gonzalez-Fernandez and R. Muñoz (Kidlington: Woodhead Publishing), 93-111.

Hamilton, J. J., and Reed, J. L. (2012). Identification of functional differences in metabolic networks using comparative genomics and constraint-based models. PLoS ONE 7:e34670. doi: 10.1371/journal.pone.0034670
Hamilton, J. J., and Reed, J. L. (2014). Software platforms to facilitate reconstructing genome-scale metabolic networks. Environ. Microbiol. 16, 49-59. doi: 10.1111/1462-2920.12312

Hashimoto, R., Ueta, R., Abe, C., Osakabe, Y., and Osakabe, K. (2018). Efficient multiplex genome editing induces precise, and self-ligated type mutations in tomato plants. Front. Plant Sci. 9:916. doi: 10.3389/fpls.2018.00916

Heidorn, T., Camsund, D., Huang, H. H., Lindberg, P., Oliveira, P., Stensjo, K., et al. (2011). Synthetic biology in cyanobacteria engineering and analyzing novel functions. Method Enzymol. 497, 539-579. doi: 10.1016/B978-0-12-385075-1.00024-X

Hendry, J. I., Prasannan, C. B., Joshi, A., Dasgupta, S., and Wangikar, P. P. (2016). Metabolic model of Synechococcus sp. PCC 7002: prediction of flux distribution and network modification for enhanced biofuel production. Bioresour. Technol. 213, 190-197. doi: 10.1016/j.biortech.2016.02.128

Hernandez-Prieto, M. A., and Futschik, M. E. (2012). CyanoEXpress: a web database for exploration and visualisation of the integrated transcriptome of cyanobacterium Synechocystis sp. PCC6803. Bioinformation 8, 634-638. doi: 10.6026/97320630008634

Herrero, A., Muro-Pastor, A. M., and Flores, E. (2001). Nitrogen control in cyanobacteria. J. Bacteriol. 183, 411-425. doi: 10.1128/JB.183.2.411-425.2001

Hirokawa, Y., Matsuo, S., Hamada, H., Matsuda, F., and Hanai, T. (2017). Metabolic engineering of Synechococcus elongatus PCC 7942 for improvement of 1,3-propanediol and glycerol production based on in silico simulation of metabolic flux distribution. Microb. Cell Fact. 16:212. doi: 10.1186/s12934-017-0824-4

Hong, S. J., and Lee, C. G. (2007). Evaluation of central metabolism based on a genomic database of Synechocystis PCC 6803. Biotechnol. Bioprocess Eng. 12, 165-173. doi: 10.1007/BF03028644

Huang, H. H., Camsund, D., Lindblad, P., and Heidorn, T. (2010). Design and characterization of molecular tools for a synthetic biology approach towards developing cyanobacterial biotechnology. Nucleic Acids Res. 38, 2577-2593. doi: $10.1093 / \mathrm{nar} / \mathrm{gkq} 164$

Huang, H. H., and Lindblad, P. (2013). Wide-dynamic-range promoters engineered for cyanobacteria. J. Biol. Eng. 7:10. doi: 10.1186/1754-1611-7-10

Immethun, C. M., DeLorenzo, D. M., Focht, C. M., Gupta, D., Johnson, C. B., and Moon, T. S. (2017). Physical, chemical, and metabolic state sensors expand the synthetic biology toolbox for Synechocystis sp. PCC 6803. Biotechnol. Bioeng. 114, 1561-1569. doi: 10.1002/bit.26275

Immethun, C. M., Ng, K. M., DeLorenzo, D. M., Waldron-Feinstein, B., Lee, Y. C., and Moon, T. S. (2016). Oxygen-responsive genetic circuits constructed in Synechocystis sp. PCC 6803. Biotechnol. Bioeng. 113, 433-442. doi: 10.1002/bit.25722

Jaiswal, D., Sengupta, A., Sohoni, S., Sengupta, S., Phadnavis, A. G., Pakrasi, H. B., et al. (2018). Genome features and biochemical characteristics of a robust, fast growing and naturally transformable cyanobacterium Synechococcus elongatus PCC 11801 isolated from India. Sci. Rep. 8:16632. doi: $10.1038 / \mathrm{s} 41598-018-34872-\mathrm{z}$

Johnson, T. J., Jahandideh, A., Isaac, I. C., Baldwin, E. L., Muthukumarappan, K., Zhou, R., et al. (2017). Determining the optimal nitrogen source for large-scale cultivation of filamentous cyanobacteria. J. Appl. Phycol. 29, 1-13. doi: 10.1007/s10811-016-0923-3

Jones, P. R. (2014). Genetic instability in cyanobacteria - an elephant in the room? Front. Bioeng. Biotechnol. 2:12. doi: 10.3389/fbioe.2014.00012

Jordan, A., Chandler, J., MacCready, J. S., Huang, J., Osteryoung, K. W., and Ducat, D. C. (2017). Engineering cyanobacterial cell morphology for enhanced recovery and processing of biomass. Appl. Environ. Microbiol. 83:AEM.0005317. doi: 10.1128/AEM.00053-17

Joshi, C. J., Peebles, C. A. M., and Prasad, A. (2017). Modeling and analysis of flux distribution and bioproduct formation in Synechocystis sp. PCC 6803 using a new genome-scale metabolic reconstruction. Algal Res. 27, 295-310. doi: 10.1016/j.algal.2017.09.013

Kämäräinen, J., Knoop, H., Stanford, N. J., Guerrero, F., Akhtar, M. K., Aro, E. M., et al. (2012). Physiological tolerance and stoichiometric potential of cyanobacteria for hydrocarbon fuel production. J. Biotechnol. 162, 67-74. doi: 10.1016/j.jbiotec.2012.07.193

Kamennaya, N. A., Ahn, S. E., Park, H., Bartal, R., Sasaki, K. A., Holman, H. Y., et al. (2015). Installing extra bicarbonate transporters in the cyanobacterium 
Synechocystis sp. PCC6803 enhances biomass production. Metabol. Eng. 29, 76-85. doi: 10.1016/j.ymben.2015.03.002

Kanehisa, M., and Goto, S. (2000). KEGG: kyoto encyclopedia of genes and genomes. Nucleic Acids Res. 28, 27-30. doi: 10.1093/nar/28.1.27

Kanno, M., Carroll, A. L., and Atsumi, S. (2017). Global metabolic rewiring for improved $\mathrm{CO}_{2}$ fixation and chemical production in cyanobacteria. Nat. Commun. 8, 1-11. doi: 10.1038/ncomms14724

Kelly, C. L., Taylor, G. M., Hitchcock, A., Torres-Mendez, A., and Heap, J. T. (2018). A rhamnose-inducible system for precise and temporal control of gene expression in cyanobacteria. ACS Synth. Biol. 7, 1056-1066. doi: 10.1021/acssynbio.7b00435

Kim, B., Kim, W. J., Kim, D. I., and Lee, S. Y. (2015). Applications of genomescale metabolic network model in metabolic engineering. J. Ind. Microbiol. Biotechnol. 42, 339-348. doi: 10.1007/s10295-014-1554-9

Kim, H. U., Charusanti, P., Lee, S. Y., and Weber, T. (2016). Metabolic engineering with systems biology tools to optimize production of prokaryotic secondary metabolites. Nat. Prod. Rep. 33, 933-941. doi: 10.1039/C6NP00019C

Kim, J., and Reed, J. L. (2010). OptORF: optimal metabolic and regulatory perturbations for metabolic engineering of microbial strains. BMC Syst. Biol. 4:53. doi: 10.1186/1752-0509-4-53

Kim, J., and Reed, J. L. (2014). Refining metabolic models and accounting for regulatory effects. Curr. Opin. Biotechnol. 29, 34-38. doi: 10.1016/j.copbio.2014.02.009

Kim, W. J., Lee, S. M., Um, Y., Sim, S. J., and Woo, H. M. (2017). Development of SyneBrick vectors as a synthetic biology platform for gene expression in Synechococcus elongatus PCC 7942. Front. Plant Sci. 8:293. doi: 10.3389/fpls.2017.00293

Klähn, S., Bolay, P., Wright, P. R., Atilho, R. M., Brewer, K. I., Hagemann, M., et al. (2018). A glutamine riboswitch is a key element for the regulation of glutamine synthetase in cyanobacteria. Nucleic Acids Res. 46, 10082-10094. doi: $10.1093 /$ nar/gky709

Klanchui, A., Khannapho, C., Phodee, A., Cheevadhanarak, S., and Meechai, A. (2012). iAK692: a genome-scale metabolic model of Spirulina platensis C1. BMC Syst. Biol. 6:71. doi: 10.1186/1752-0509-6-71

Knoop, H., Gründel, M., Zilliges, Y., Lehmann, R., Hoffmann, S., Lockau, W., et al. (2013). Flux balance analysis of cyanobacterial metabolism: the metabolic network of Synechocystis sp. PCC 6803. PLoS Comput. Biol. 9:e1003081. doi: 10.1371/journal.pcbi.1003081

Knoop, H., Zilliges, Y., Lockau, W., and Steuer, R. (2010). The metabolic network of Synechocystis sp. PCC 6803: systemic properties of autotrophic growth. Plant Physiol. 154, 410-422. doi: 10.1104/pp.110.157198

Knoot, C. J., Ungerer, J., Wangikar, P. P., and Pakrasi, H. B. (2018). Cyanobacteria: promising biocatalysts for sustainable chemical production. J. Biol. Chem. 293, 5044-5052. doi: 10.1074/jbc.R117.815886

Kojima, K., Keta, S., Uesaka, K., Kato, A., Takatani, N., Ihara, K., et al. (2016). A simple method for isolation and construction of markerless cyanobacterial mutants defective in acyl-acyl carrier protein synthetase. Appl. Microbiol. Biotechnol. 100, 10107-10113. doi: 10.1007/s00253-016-7850-8

Koksharova, O. A., and Wolk, C. P. (2002). Genetic tools for cyanobacteria. Appl. Microbiol. Biotechnol. 58, 123-137. doi: 10.1007/s00253-001-0864-9

Kopka, J., Schmidt, S., Dethloff, F., Pade, N., Berendt, S., Schottkowski, M., et al. (2017). Systems analysis of ethanol production in the genetically engineered cyanobacterium Synechococcus sp. PCC 7002. Biotechnol. Biofuels 10:56. doi: 10.1186/s13068-017-0741-0

Krapp, A., Quick, W. P., and Stitt, M. (1991). Ribulose-1,5-bisphosphate carboxylase-oxygenase, other Calvin-cycle enzymes, and chlorophyll decrease when glucose is supplied to mature spinach leaves via the transpiration stream. Planta 186, 58-69. doi: 10.1007/BF00201498

Krishnakumar, S., Durai, D. A., Wangikar, P. P., and Viswanathan, G. A. (2013). SHARP: genome-scale identification of gene-protein-reaction associations in cyanobacteria. Photosyn. Res. 118, 181-190. doi: 10.1007/s11120-0139910-6

Kumar, A., Suthers, P. F., and Maranas, C. D. (2012). MetRxn: a knowledgebase of metabolites and reactions spanning metabolic models and databases. BMC Bioinformatics 13:6. doi: 10.1186/1471-2105-13-6

Kumar, K., Mella-Herrera, R. A., and Golden, J. W. (2010). Cyanobacterial heterocysts. Cold Spring Harb. Perspect. Biol. 2:a000315. doi: $10.1101 /$ cshperspect.a000315
Kumar, R. R., and Prasad, S. (2011). Metabolic engineering of bacteria. Indian J. Microbiol. 51, 403-409. doi: 10.1007/s12088-011-0172-8

Kunst, F., and Rapoport, G. (1995). Salt stress is an environmental signal affecting degradative enzyme synthesis in Bacillus subtilis. J. Bacteriol. 177, 2403-2407. doi: 10.1128/jb.177.9.2403-2407.1995

Kuritz, T., and Wolk, C. P. (1995). Use of filamentous cyanobacteria for biodegradation of organic pollutants. Appl. Environ. Microbiol. 61:1169.

Lai, M. C., and Lan, E. I. (2015). Advances in metabolic engineering of cyanobacteria for photosynthetic biochemical production. Metabolites 5, 636-658. doi: 10.3390/metabo5040636

Landry, B. P., Stöckel, J., and Pakrasi, H. B. (2013). Use of degradation tags to control protein levels in the Cyanobacterium Synechocystis sp. strain PCC 6803. Appl. Environ. Microbiol. 79, 2833-2835. doi: 10.1128/AEM.03741-12

Lau, N. S., Matsui, M., and Abdullah, A. A. (2015). Cyanobacteria: photoautotrophic microbial factories for the sustainable synthesis of industrial products. Biomed Res. Int. 2015:754934. doi: 10.1155/2015/754934

Lee, J. W., Kim, T. Y., Jang, Y. S., Choi, S., and Lee, S. Y. (2011). Systems metabolic engineering for chemicals and materials. Trends Biotechnol. 29, 370-378. doi: 10.1016/j.tibtech.2011.04.001

Lewis, N. E., Nagarajan, H., and Palsson, B. O. (2012). Constraining the metabolic genotype-phenotype relationship using a phylogeny of in silico methods. Nat. Rev. Microbiol. 10, 291-305. doi: 10.1038/nrmicro2737

Li, H., Shen, C. R., Huang, C. H., Sung, L. Y., Wu, M. Y., and Hu, Y. C. (2016) CRISPR-Cas9 for the genome engineering of cyanobacteria and succinate production. Metab. Eng. 38, 293-302. doi: 10.1016/j.ymben.2016.09.006

Li, X., Shen, C. R., and Liao, J. C. (2014). Isobutanol production as an alternative metabolic sink to rescue the growth deficiency of the glycogen mutant of Synechococcus elongatus PCC 7942. Photosyn. Res. 120, 301-310. doi: 10.1007/s11120-014-9987-6

Lian, J., Bao, Z., Hu, S., and Zhao, H. (2018). Engineered CRISPR/Cas9 system for multiplex genome engineering of polyploid industrial yeast strains. Biotechnol. Bioeng. 115, 1630-1635. doi: 10.1002/bit.26569

Liang, F., Lindberg, P., and Lindblad, P. (2018). Engineering photoautotrophic carbon fixation for enhanced growth and productivity. Sustain. Ener. Fuels. 2, 2583-2600. doi: 10.1039/C8SE00281A

Liang, F., and Lindblad, P. (2016). Effects of overexpressing photosynthetic carbon flux control enzymes in the cyanobacterium Synechocystis PCC 6803. Metab. Eng. 38, 56-64. doi: 10.1016/j.ymben.2016. 06.005

Lin, P. C., Saha, R., Zhang, F., and Pakrasi, H. B. (2017). Metabolic engineering of the pentose phosphate pathway for enhanced limonene production in the cyanobacterium Synechocystis sp. PCC 6803. Sci. Rep. 7:17503. doi: 10.1038/s41598-017-17831-y

Liu, D., and Pakrasi, H. B. (2018). Exploring native genetic elements as plug-in tools for synthetic biology in the cyanobacterium Synechocystis sp. PCC 6803. Microb. Cell Fact. 17:48. doi: 10.1186/s12934-018-0897-8

Liu, Y., Tsinoremas, N. F., Johnson, C. H., Lebedeva, N. V., Golden, S. S., Ishiura, M., et al. (1995). Circadian orchestration of gene expression in cyanobacteria. Genes Dev. 9, 1469-1478. doi: 10.1101/gad.9.12.1469

Lucker, B. F., Hall, C. C., Zegarac, R., and Kramer, D. M. (2014). The environmental photobioreactor (ePBR): an algal culturing platform for simulating dynamic natural environments. Algal Res. 6, 242-249. doi: 10.1016/j.algal.2013.12.007

Ludwig, M., and Bryant, D. A. (2012). Synechococcus sp. strain PCC 7002 transcriptome: acclimation to temperature, salinity, oxidative stress, and mixotrophic growth conditions. Front. Microbiol. 3:354. doi: 10.3389/fmicb.2012.00354

Lv, Q., Ma, W., Liu, H., Li, J., Wang, H., Lu, F., et al. (2015). Genome-wide proteinprotein interactions and protein function exploration in cyanobacteria. Sci. Rep. 5:15519. doi: 10.1038/srep15519

Ma, A. T., Schmidt, C. M., and Golden, J. W. (2014). Regulation of gene expression in diverse cyanobacterial species by using theophyllineresponsive riboswitches. Appl. Environ. Microb. 80, 6704-6713. doi: 10.1128/AEM.01697-14

Machado, D., Andrejev, S., Tramontano, M., and Patil, K. R. (2018). Fast automated reconstruction of genome-scale metabolic models for microbial species and communities. Nucleic Acids Res. 46, 7542-7553. doi: 10.1093/nar/gky537

Machado, D., Herrgård, M. J., and Rocha, I. (2016). Stoichiometric representation of gene-protein-reaction associations leverages constraint-based analysis from 
reaction to gene-level phenotype prediction. PLoS Comput. Biol. 12:e1005140. doi: 10.1371/journal.pcbi.1005140

Maia, P., Rocha, M., and Rocha, I. (2016). In silico constraint-based strain optimization methods: the quest for optimal cell factories. Microbiol. Mol. Biol. Rev. 80, 45-67. doi: 10.1128/MMBR.00014-15

Malatinszky, D., Steuer, R., and Jones, P. R. (2017). A comprehensively curated genome-scale two-cell model for the heterocystous cyanobacterium Anabaena sp. PCC 7120. Plant Physiol. 173, 509-523. doi: 10.1104/pp.16.01487

Markley, A. L., Begemann, M. B., Clarke, R. E., Gordon, G. C., and Pfleger, B. F. (2015). Synthetic biology toolbox for controlling gene expression in the cyanobacterium Synechococcus sp. strain PCC 7002. ACS Synth. Biol. 4, 595-603. doi: 10.1021/sb500260k

Markson, J. S., Piechura, J. R., Puszynska, A. M., and O’Shea, E. K. (2013). Circadian control of global gene expression by the cyanobacterial master regulator RpaA. Cell 155, 1396-1408. doi: 10.1016/j.cell.2013.11.005

Marraccini, P., Bulteau, S., Cassier-Chauvat, C., Mermet-Bouvier, P., and Chauvat, F. (1993). A conjugative plasmid vector for promoter analysis in several cyanobacteria of the genera Synechococcus and Synechocystis. Plant Mol. Biol. 23, 905-909. doi: 10.1007/BF00021546

Matsuoka, M., Takahama, K., and Ogawa, T. (2001). Gene replacement in cyanobacteria mediated by a dominant streptomycin-sensitive rps 12 gene that allows selection of mutants free from drug resistance markers. Microbiology 147, 2077-2087. doi: 10.1099/00221287-147-8-2077

Megharaj, M., Venkateswarlu, K., and Rao, A. S. (1987). Metabolism of monocrotophos and quinalphos by algae isolated from soil. Bull. Environ. Contam. Toxicol. 39, 251-256. doi: 10.1007/BF01689414

Mohammadi, R., Fallah-Mehrabadi, J., Bidkhori, G., Zahiri, J., Javad Niroomand, M., and Masoudi-Nejad, A. (2016). A systems biology approach to reconcile metabolic network models with application to Synechocystis sp. PCC 6803 for biofuel production. Mol. Biosyst. 12, 2552-2561. doi: 10.1039/C6MB00119J

Montagud, A., Navarro, E., Fernández de Córdoba, P., Urchueguía, J. F., and Patil, K. R. (2010). Reconstruction and analysis of genome-scale metabolic model of a photosynthetic bacterium. BMC Syst. Biol. 4:156. doi: 10.1186/1752-05094-156

Montagud, A., Zelezniak, A., Navarro, E., de Cordoba, P. F., Urchueguia, J. F., and Patil, K. R. (2011). Flux coupling and transcriptional regulation within the metabolic network of the photosynthetic bacterium Synechocystis sp. PCC 6803. Biotechnol. J. 6, 330-342. doi: 10.1002/biot.2010 00109

Mori, T., Binder, B., and Johnson, C. H. (1996). Circadian gating of cell division in cyanobacteria growing with average doubling times of less than 24 hours. Proc. Natl. Acad. Sci. U.S.A. 93, 10183-10188. doi: 10.1073/pnas.93.19. 10183

Mueller, T. J., Berla, B. M., Pakrasi, H. B., and Maranas, C. D. (2013). Rapid construction of metabolic models for a family of Cyanobacteria using a multiple source annotation workflow. BMC Syst. Biol. 7:142. doi: 10.1186/1752-0509-7-142

Mueller, T. J., Ungerer, J. L., Pakrasi, H. B., and Maranas, C. D. (2017). Identifying the metabolic differences of a fast-growth phenotype in Synechococcus UTEX 2973. Sci. Rep. 7:41569. doi: 10.1038/srep41569

Mutalik, V. K., Guimaraes, J. C., Cambray, G., Lam, C., Christoffersen, M. J., Mai, Q. A., et al. (2013). Precise and reliable gene expression via standard transcription and translation initiation elements. Nat. Methods 10, 354-360. doi: 10.1038/nmeth.2404

Nakahira, Y., Ogawa, A., Asano, H., Oyama, T., and Tozawa, Y. (2013). Theophylline-dependent riboswitch as a novel genetic tool for strict regulation of protein expression in Cyanobacterium Synechococcus elongatus PCC 7942. Plant Cell Physiol. 54, 1724-1735. doi: 10.1093/pcp/pct115

Narro, M. L., Cerniglia, C. E., Van Baalen, C., and Gibson, D. T. (1992). Evidence for an NIH shift in oxidation of naphthalene by the marine cyanobacterium Oscillatoria sp. strain JCM. Appl. Environ. Microbiol. 58, 1360-1363.

National Academies of Sciences, Engineering and Medicine (2018). Gaseous Carbon Waste Streams Utilization: Status and Research Needs. Washington, DC: The National Academies Press.

Niu, T. C., Lin, G. M., Xie, L. R., Wang, Z. Q., Xing, W. Y., Zhang, J. Y., et al. (2018). Expanding the potential of CRISPR-Cpf1 based genome editing technology in the cyanobacterium Anabaena PCC 7120. ACS Synth. Biol. 8, 170-180. doi: 10.1021/acssynbio.8b00437
Nogales, J., Gudmundsson, S., Duque, E., Ramos, J. L., and Palsson, B. O. (2017). Expanding the computable reactome in Pseudomonas putida reveals metabolic cycles providing robustness. bioRxiv. doi: 10.1101/139121

Nogales, J., Gudmundsson, S., Knight, E. M., Palsson, B. O., and Thiele, I. (2012). Detailing the optimality of photosynthesis in cyanobacteria through systems biology analysis. Proc. Natl. Acad. Sci. U.S.A. 109, 2678-2683. doi: 10.1073/pnas.1117907109

Nogales, J., Gudmundsson, S., and Thiele, I. (2013). Toward systems metabolic engineering in cyanobacteria: opportunities and bottlenecks. Bioengineered 4, 158-163. doi: 10.4161/bioe.22792

Nogales, J., Palsson, B. O., and Thiele, I. (2008). A genome-scale metabolic reconstruction of Pseudomonas putida KT2440: iJN746 as a cell factory. BMC Syst. Biol. 2:79. doi: 10.1186/1752-0509-2-79

Noguchi, T., and Golden, S. S. (2017). Bioluminescent and Fluorescent Reporters in Circadian Rhythm Studies, 1-24. Available online at: https://ccb.ucsd.edu/thebioclock-studio/education-resources/reporter-review/ReporterReviewPDF. pdf (Accessed October 15, 2018).

Nozzi, N. E., Oliver, J. W., and Atsumi, S. (2013). Cyanobacteria as a platform for biofuel production. Front. Bioeng. Biotechnol. 1:7. doi: 10.3389/fbioe.2013.00007

Nudler, E. (2006). Flipping riboswitches. Cell 126, 19-22. doi: $10.1016 /$ j.cell.2006.06.024

O'Brien, E. J., Monk, J. M., and Palsson, B. O. (2015). Using genomescale models to predict biological capabilities. Cell 161, 971-987. doi: 10.1016/j.cell.2015.05.019

Ohbayashi, R., Akai, H., Yoshikawa, H., Hess, W. R., and Watanabe, S. (2016). A tightly inducible riboswitch system in Synechocystis sp. PCC 6803. J. Gen. Appl. Microbiol. 62, 154-159. doi: 10.2323/jgam.2016.02.002

Oliver, J. W., Machado, I. M., Yoneda, H., and Atsumi, S. (2013). Cyanobacterial conversion of carbon dioxide to 2,3-butanediol. Proc. Natl. Acad. Sci. U.S.A. 110, 1249-1254. doi: 10.1073/pnas.1213024110

Orth, J. D., Conrad, T. M., Na, J., Lerman, J. A., Nam, H., Feist, A. M., et al. (2011). A comprehensive genome-scale reconstruction of Escherichia coli metabolism. Mol. Syst. Biol. 7:535. doi: 10.1038/msb.2011.65

Orth, J. D., Thiele, I., and Palsson, B. O. (2010). What is flux balance analysis? Nat. Biotechnol. 28, 245-248. doi: 10.1038/nbt.1614

Overbeek, R., Olson, R., Pusch, G. D., Olsen, G. J., Davis, J. J., Disz, T., et al. (2014). The SEED and the Rapid Annotation of microbial genomes using Subsystems Technology (RAST). Nucleic Acids Res. 42(Database issue), D206D214. doi: 10.1093/nar/gkt1226

Parmar, A., Singh, N. K., Pandey, A., Gnansounou, E., and Madamwar, D. (2011). Cyanobacteria and microalgae: a positive prospect for biofuels. Bioresour. Technol. 102, 10163-10172. doi: 10.1016/j.biortech.2011.08.030

Pasotti, L., Politi, N., Zucca, S., Cusella De Angelis, M. G., and Magni, P. (2012). Bottom-up engineering of biological systems through standard bricks: a modularity study on basic parts and devices. PLoS ONE 7:e39407. doi: 10.1371/annotation/91e7d3a1-2f50-4f84-8b12-2c21f88438c3

Pathak, J., Rajneesh, M. P. K., Singh, S. P., Häder, D. P., and Sinha, R. P. (2018). Cyanobacterial farming for environment friendly sustainable agriculture practices: innovations and perspectives. Front. Environ. Sci. 6:7. doi: 10.3389/fenvs.2018.00007

Patil, K. R., Rocha, I., Förster, J., and Nielsen, J. (2005). Evolutionary programming as a platform for in silico metabolic engineering. BMC Bioinformatics 6:308. doi: 10.1186/1471-2105-6-308

Paul, M. J., and Foyer, C. H. (2001). Sink regulation of photosynthesis. J. Exp. Bot. 52, 1383-1400. doi: 10.1093/jexbot/52.360.1383

Pédelacq, J. D., Cabantous, S., Tran, T., Terwilliger, T. C., and Waldo, G. S. (2006). Engineering and characterization of a superfolder green fluorescent protein. Nat. Biotechnol. 24, 79-88. doi: 10.1038/nbt1172

Pérez, A. A., Rodionov, D. A., and Bryant, D. A. (2016). Identification and regulation of genes for cobalamin transport in the Cyanobacterium Synechococcus sp. strain PCC 7002. J. Bacteriol. 198, 2753-2761. doi: 10.1128/JB.00476-16

Popp, P. F., Dotzler, M., Radeck, J., Bartels, J., and Mascher, T. (2017). The Bacillus BioBrick Box 2.0: expanding the genetic toolbox for the standardized work with Bacillus subtilis. Sci. Rep. 7:15058. doi: 10.1038/s41598-017-15107-z

Porter, R. D. (1986). Transformation in cyanobacteria. Crit. Rev. Microbiol. 13, 111-132. doi: 10.3109/10408418609108736 
Price, G. D., Badger, M. R., and Von Caemmerer, S. (2011). The prospect of using cyanobacterial bicarbonate transporters to improve leaf photosynthesis in C3 crop plants. Plant Physiol. 155, 20-26. doi: 10.1104/pp.110.164681

Price, G. D., Badger, M. R., Woodger, F. J., and Long, B. M. (2008). Advances in understanding the cyanobacterial $\mathrm{CO}_{2}$-concentrating-mechanism (CCM): functional components, $\mathrm{Ci}$ transporters, diversity, genetic regulation and prospects for engineering into plants. J. Exp. Bot. 59, 1441-1461. doi: $10.1093 / \mathrm{jxb} / \mathrm{erm} 112$

Qian, X., Kim, M. K., Kumaraswamy, G. K., Agarwal, A., Lun, D. S., and Dismukes, G. C. (2017). Flux balance analysis of photoautotrophic metabolism: uncovering new biological details of subsystems involved in cyanobacterial photosynthesis. Biochim. Biophys. Acta Bioenerg. 1858, 276-287. doi: 10.1016/j.bbabio.2016.12.007

Quintana, N., Van der Kooy, F., Van de Rhee, M. D., Voshol, G. P., and Verpoorte, R. (2011). Renewable energy from Cyanobacteria: energy production optimization by metabolic pathway engineering. Appl. Microbiol. Biotechnol. 91, 471-490. doi: 10.1007/s00253-011-3394-0

Raman, K., and Chandra, N. (2009). Flux balance analysis of biological systems: applications and challenges. Brief. Bioinformatics 10, 435-449. doi: 10.1093/bib/bbp011

Ramey, C. J., Baron-Sola, A., Aucoin, H. R., and Boyle, N. R. (2015). Genome engineering in cyanobacteria: where we are and where we need to go. ACS Synth. Biol. 4, 1186-1196. doi: 10.1021/acssynbio.5b00043

Ranganathan, S., Suthers, P. F., and Maranas, C. D. (2010). OptForce: an optimization procedure for identifying all genetic manipulations leading to targeted overproductions. PLoS Comput. Biol. 6:e1000744. doi: 10.1371/journal.pcbi.1000744

Reed, J. L., Vo, T. D., Schilling, C. H., and Palsson, B. O. (2003). An expanded genome-scale model of Escherichia coli K-12 (iJR904 GSM/GPR). Genome Biol. 4:R54. doi: 10.1186/gb-2003-4-9-r54

Ren, Q., Kang, K. H., and Paulsen, I. T. (2004). TransportDB: a relational database of cellular membrane transport systems. Nucleic Acids Res. 32(Database issue), D284-D288. doi: 10.1093/nar/gkh016

Rodriguez, E. A., Campbell, R. E., Lin, J. Y., Lin, M. Z., Miyawaki, A., Palmer, A. E., et al. (2017). The growing and glowing toolbox of fluorescent and photoactive proteins. Trends Biochem. Sci. 42, 111-129. doi: 10.1016/j.tibs.2016. 09.010

Rubin, B. E., Wetmore, K. M., Price, M. N., Diamond, S., Shultzaberger, R. K., Lowe, L. C., et al. (2015). The essential gene set of a photosynthetic organism. Proc. Natl. Acad. Sci. U.S.A. 112, E6634-E6643. doi: 10.1073/pnas.15192 20112

Ruffing, A. M. (2011). Engineered cyanobacteria: teaching an old bug new tricks. Bioeng. Bugs 2, 136-149. doi: 10.4161/bbug.2.3.15285

Ruffing, A. M. (2014). Improved free fatty acid production in cyanobacteria with Synechococcus sp. PCC 7002 as host. Front. Bioeng. Biotechnol. 2:17. doi: 10.3389/fbioe.2014.00017

Ruffing, A. M., Jensen, T. J., and Strickland, L. M. (2016). Genetic tools for advancement of Synechococcus sp. PCC 7002 as a cyanobacterial chassis. Microb. Cell Fact. 15:190. doi: 10.1186/s12934-016-0584-6

Saha, R., Verseput, A. T., Berla, B. M., Mueller, T. J., Pakrasi, H. B., and Maranas, C. D. (2012). Reconstruction and comparison of the metabolic potential of cyanobacteria Cyanothece sp. ATCC 51142 and Synechocystis sp. PCC 6803. PLoS ONE 7:e48285. doi: 10.1371/journal.pone.0048285

Sakuma, T., Nishikawa, A., Kume, S., Chayama, K., and Yamamoto, T. (2014). Multiplex genome engineering in human cells using all-in-one CRISPR/Cas9 vector system. Sci. Rep. 4:5400. doi: 10.1038/srep05400

Salis, H. M. (2011). The ribosome binding site calculator. Method Enzymol. 498, 19-42. doi: 10.1016/B978-0-12-385120-8.00002-4

Salvador-Reyes, L. A., and Luesch, H. (2015). Biological targets and mechanisms of action of natural products from marine cyanobacteria. Nat. Prod. Rep. 32, 478-503. doi: 10.1039/C4NP00104D

Santos-Merino, M., Garcillán-Barcia, M. P., and de la Cruz, F. (2018). Engineering the fatty acid synthesis pathway in Synechococcus elongatus PCC 7942 improves omega-3 fatty acid production. Biotechnol. Biofuels 11:239. doi: 10.1186/s13068-018-1243-4

Sawada, S., Hayakawa, T., Fukushi, K., and Kasai, M. (1986). Influence of carbohydrates on photosynthesis in single, rooted soybean leaves used as a source-sink model. Plant Cell Physiol. 27, 591-600.
Schleif, R. (2010). AraC protein, regulation of the l-arabinose operon in Escherichia coli, and the light switch mechanism of AraC action. FEMS Microbiol. Rev. 34, 779-796. doi: 10.1111/j.1574-6976.2010.00226.x

Schoepp, N. G., Stewart, R. L., Sun, V., Quigley, A. J., Mendola, D., Mayfield, S. P., et al. (2014). System and method for research-scale outdoor production of microalgae and cyanobacteria. Bioresour. Technol. 166, 273-281. doi: 10.1016/j.biortech.2014.05.046

Schomburg, I., Chang, A., and Schomburg, D. (2002). BRENDA, enzyme data and metabolic information. Nucleic Acids Res. 30, 47-49. doi: 10.1093/nar/30.1.47

Segrè, D., Vitkup, D., and Church, G. M. (2002). Analysis of optimality in natural and perturbed metabolic networks. Proc. Natl. Acad. Sci. U.S.A. 99, 15112-15117. doi: 10.1073/pnas.232349399

Sengupta, A., Pakrasi, H. B., and Wangikar, P. P. (2018). Recent advances in synthetic biology of cyanobacteria. Appl. Microbiol. Biotechnol. 102, 5457-5471. doi: 10.1007/s00253-018-9046-x

Shabestary, K., and Hudson, E. P. (2016). Computational metabolic engineering strategies for growth-coupled biofuel production by Synechocystis. Metab. Eng. Commun. 3, 216-226. doi: 10.1016/j.meteno.2016.07.003

Sharkey, T. D., Stitt, M., Heineke, D., Gerhardt, R., Raschke, K., and Heldt, H. W. (1986). Limitation of photosynthesis by carbon metabolism: II. O(2)-insensitive $\mathrm{CO}(2)$ uptake results from limitation of triose phosphate utilization. Plant Physiol. 81, 1123-1129. doi: 10.1104/pp.81.4.1123

Shastri, A. A., and Morgan, J. A. (2005). Flux balance analysis of photoautotrophic metabolism. Biotechnol. Prog. 21, 1617-1626. doi: 10.1021/bp050246d

Sheehan, J., Dunahay, T., Benemann, J., and Roessler, P. (1998). A Look Back at the U.S. Department of Energy's Aquatic Species Program: Biodiesel From Algae. Available online at: https://www.nrel.gov/docs/legosti/fy98/24190.pdf (Accessed October 15, 2018).

Sheng, J., Kim, H. W., Badalamenti, J. P., Zhou, C., Sridharakrishnan, S., Krajmalnik-Brown, R., et al. (2011). Effects of temperature shifts on growth rate and lipid characteristics of Synechocystis sp. PCC6803 in a bench-top photobioreactor. Bioresource Technology 102, 11218-11225. doi: 10.1016/j.biortech.2011.09.083

Shestakov, S. V., and Khyen, N. T. (1970). Evidence for genetic transformation in blue-green alga Anacystis nidulans. MGG Mol. Gen. Genet. 107, 372-375. doi: $10.1007 /$ BF00441199

Shetty, R. P., Endy, D., and Knight, T. F. Jr. (2008). Engineering BioBrick vectors from BioBrick parts. J. Biol. Eng. 2:5. doi: 10.1186/17541611-2-5

Shih, P. M. (2015). Cyanobacterial evolution: fresh insight into ancient questions. Curr. Biol. 25, R192-R193. doi: 10.1016/j.cub.2014.12.046

Shirai, T., Osanai, T., and Kondo, A. (2016). Designing intracellular metabolism for production of target compounds by introducing a heterologous metabolic reaction based on a Synechosystis sp. 6803 genome-scale model. Microb. Cell Fact. 15:13. doi: 10.1186/s12934-016-0416-8

Singh, J. S., Kumar, A., Rai, A. N., and Singh, D. P. (2016). Cyanobacteria: a precious bio-resource in agriculture, ecosystem, and environmental sustainability. Front. Microbiol. 7:529. doi: 10.3389/fmicb.2016.00529

Song, K., Tan, X., Liang, Y., and Lu, X. (2016). The potential of Synechococcus elongatus UTEX 2973 for sugar feedstock production. Appl. Microbiol. Biotechnol. 100, 7865-7875. doi: 10.1007/s00253-016-7510-z

Srinivasan, S., Cluett, W. R., and Mahadevan, R. (2015). Constructing kinetic models of metabolism at genome-scales: a review. Biotechnol. J. 10, 1345-1359. doi: 10.1002/biot.201400522

Stanier, R. Y., Kunisawa, R., Mandel, M., and Cohen-Bazire, G. (1971). Purification and properties of unicellular blue-green algae (order Chroococcales). Bacteriol. Rev. 35, 171-205.

Stephanopoulos, G. (2012). Synthetic biology and metabolic engineering. ACS Synth. Biol. 1, 514-525. doi: 10.1021/sb300094q

Steuer, R., Knoop, H., and Machne, R. (2012). Modelling cyanobacteria: from metabolism to integrative models of phototrophic growth. J. Exp. Bot. 63, 2259-2274. doi: 10.1093/jxb/ers018

Stevens, S. E., and Porter, R. D. (1980). Transformation in Agmenellum quadruplicatum. Proc. Natl. Acad. Sci. U.S.A. 77, 6052-6056. doi: $10.1073 /$ pnas.77.10.6052

Stucken, K., Koch, R., and Dagan, T. (2013). Cyanobacterial defense mechanisms against foreign DNA transfer and their impact on genetic engineering. Biol. Res. 46, 373-382. doi: 10.4067/S0716-97602013000400009 
Sun, T., Li, S., Song, X., Diao, J., Chen, L., and Zhang, W. (2018). Toolboxes for cyanobacteria: recent advances and future direction. Biotechnol. Adv. 36, 1293-1307. doi: 10.1016/j.biotechadv.2018.04.007

Suthers, P. F., Zomorrodi, A., and Maranas, C. D. (2009). Genome-scale gene/reaction essentiality and synthetic lethality analysis. Mol. Syst. Biol. 5:301. doi: $10.1038 / \mathrm{msb} .2009 .56$

Swarts, D. C., and Jinek, M. (2018). Cas9 versus Cas12a/Cpf1: structure-function comparisons and implications for genome editing. Wiley Interdiscip Rev. RNA 9:e1481. doi: 10.1002/wrna.1481

Takahama, K., Matsuoka, M., Nagahama, K., and Ogawa, T. (2004). Highfrequency gene replacement in cyanobacteria using a heterologous rps12 gene. Plant Cell Physiol. 45, 333-339. doi: 10.1093/pcp/pch041

Tamagnini, P., Axelsson, R., Lindberg, P., Oxelfelt, F., Wunschiers, R., and Lindblad, P. (2002). Hydrogenases and hydrogen metabolism of cyanobacteria. Microbiol. Mol. Biol. Rev. 66, 1-20. doi: 10.1128/MMBR.66.1.1-20.2002

Tan, X., Hou, S., Song, K., Georg, J., Klähn, S., Lu, X., et al. (2018). The primary transcriptome of the fast-growing cyanobacterium Synechococcus elongatus UTEX 2973. Biotechnol. Biofuels 11:218. doi: 10.1186/s13068-018-1215-8

Taton, A., Lis, E., Adin, D. M., Dong, G., Cookson, S., Kay, S. A., et al. (2012). Gene transfer in Leptolyngbya sp. strain BL0902, a cyanobacterium suitable for production of biomass and bioproducts. PLOS ONE 7:e30901. doi: 10.1371/journal.pone.0030901

Taton, A., Ma, A. T., Ota, M., Golden, S. S., and Golden, J. W. (2017). NOT gate genetic circuits to control gene expression in cyanobacteria. ACS Synth. Biol. 6, 2175-2182. doi: 10.1021/acssynbio.7b00203

Taton, A., Unglaub, F., Wright, N. E., Zeng, W. Y., Paz-Yepes, J., Brahamsha, B., et al. (2014). Broad-host-range vector system for synthetic biology and biotechnology in cyanobacteria. Nucleic Acids Res. 42:e136. doi: 10.1093/nar/gku673

Thiel, K., Mulaku, E., Dandapani, H., Nagy, C., Aro, E. M., and Kallio, P. (2018). Translation efficiency of heterologous proteins is significantly affected by the genetic context of RBS sequences in engineered cyanobacterium Synechocystis sp. PCC 6803. Microb. Cell Fact. 17:34. doi: 10.1186/s12934-018-0882-2

Thiel, T., and Poo, H. (1989). Transformation of a filamentous cyanobacterium by electroporation. J. Bacteriol. 171, 5743-5746. doi: $10.1128 /$ jb.171.10.5743-5746.1989

Thiele, I., and Palsson, B. O. (2010). A protocol for generating a highquality genome-scale metabolic reconstruction. Nat. Protoc. 5, 93-121. doi: 10.1038/nprot.2009.203

Thomas, A., Rahmanian, S., Bordbar, A., Palsson, B. O., and Jamshidi, N. (2014). Network reconstruction of platelet metabolism identifies metabolic signature for aspirin resistance. Sci. Rep. 4:3925. doi: 10.1038/ srep03925

Triana, J., Montagud, A., Siurana, M., Fuente, D., Urchueguia, A., Gamermann, D., et al. (2014). Generation and evaluation of a genome-scale metabolic network model of Synechococcus elongatus PCC 7942. Metabolites 4, 680-698. doi: 10.3390/metabo4030680

Tsinoremas, N. F., Kutach, A. K., Strayer, C. A., and Golden, S. S. (1994). Efficient gene transfer in Synechococcus sp. strains PCC 7942 and PCC 6301 by interspecies conjugation and chromosomal recombination. J. Bacteriol. 176, 6764-6768. doi: 10.1128/jb.176.21.6764-6768.1994

Ungerer, J., and Pakrasi, H. B. (2016). Cpf1 is a versatile tool for CRISPR genome editing across diverse species of cyanobacteria. Sci. Rep. 6:39681. doi: $10.1038 /$ srep39681

Ungerer, J., Tao, L., Davis, M., Ghirardi, M., Maness, P. C., and Yu, J. (2012). Sustained photosynthetic conversion of $\mathrm{CO}_{2}$ to ethylene in recombinant cyanobacterium Synechocystis 6803. Energy Environ. Sci. 5, 8998-9006. doi: $10.1039 /$ c2ee22555g

Ungerer, J., Wendt, K. E., Hendry, J. I., Maranas, C. D., and Pakrasi, H. B. (2018). Comparative genomics reveals the molecular determinants of rapid growth of the cyanobacterium Synechococcus elongatus UTEX 2973. Proc. Natl. Acad. Sci. U.S.A. 115, E11761-E11770. doi: 10.1073/pnas.1814912115

Utschig, L. M., Silver, S. C., Mulfort, K. L., and Tiede, D. M. (2011). Naturedriven photochemistry for catalytic solar hydrogen production: a Photosystem I-transition metal catalyst hybrid. J. Am. Chem. Soc. 133, 16334-16337. doi: $10.1021 /$ ja206012r
Vasudevan, R., Gale, G. A. R., Schiavon, A. A., Puzorjov, A., Malin, J., Gillespie, M. D., et al. (2018). CyanoGate: a golden gate modular cloning suite for engineering cyanobacteria based on the Plant MoClo syntax. bioRxiv. doi: $10.1101 / 426700$

Vermaas, W. (1996). Molecular genetics of the cyanobacterium Synechocystis sp. PCC 6803: principles and possible biotechnology applications. J. Appl. Phycol. 8, 263-273. doi: 10.1007/BF02178569

Vermass, W. F., Rutherford, A. W., and Hansson, O. (1988). Site-directed mutagenesis in photosystem II of the cyanobacterium Synechocystis sp. PCC 6803: donor D is a tyrosine residue in the D2 protein. Proc. Natl. Acad. Sci. U.S.A. 85, 8477-8481. doi: 10.1073/pnas.85.22.8477

Viola, S., Rühle, T., and Leister, D. (2014). A single vector-based strategy for marker-less gene replacement in Synechocystis sp. PCC 6803. Microb. Cell Fact. 13:4. doi: 10.1186/1475-2859-13-4

Vitkin, E., and Shlomi, T. (2012). MIRAGE: a functional genomicsbased approach for metabolic network model reconstruction and its application to cyanobacteria networks. Genome Biol. 13:R111. doi: $10.1186 / \mathrm{gb}-2012-13-11-\mathrm{r} 111$

Vivek-Ananth, R. P., and Samal, A. (2016). Advances in the integration of transcriptional regulatory information into genome-scale metabolic models. BioSystems 147, 1-10. doi: 10.1016/j.biosystems.2016.06.001

Vogel, A. I. M., Lale, R., and Hohmann-Marriott, M. F. (2017). Streamlining recombination-mediated genetic engineering by validating three neutral integration sites in Synechococcus sp. PCC 7002. J. Biol. Eng. 11:19. doi: 10.1186/s13036-017-0061-8

Vu, T. T., Hill, E. A., Kucek, L. A., Konopka, A. E., Beliaev, A. S., and Reed, J. L. (2013). Computational evaluation of Synechococcus sp. PCC 7002 metabolism for chemical production. Biotechnol. J. 8, 619-630. doi: 10.1002/biot.201200315

Vu, T. T., Stolyar, S. M., Pinchuk, G. E., Hill, E. A., Kucek, L. A., Brown, R. N., et al. (2012). Genome-scale modeling of light-driven reductant partitioning and carbon fluxes in diazotrophic unicellular cyanobacterium Cyanothece sp. ATCC 51142. PLoS Comput. Biol. 8:e1002460. doi: 10.1371/journal.pcbi.1002460

Wang, B., Eckert, C., Maness, P. C., and Yu, J. (2018). A genetic toolbox for modulating the expression of heterologous genes in the Cyanobacterium Synechocystis sp. PCC 6803. ACS Synth. Biol. 7, 276-286. doi: 10.1021/acssynbio.7b00297

Wang, B., Wang, J., Zhang, W., and Meldrum, D. R. (2012). Application of synthetic biology in cyanobacteria and algae. Front. Microbiol. 3:344. doi: $10.3389 /$ fmicb. 2012.00344

Wang, H. H., Isaacs, F. J., Carr, P. A., Sun, Z. Z., Xu, G., Forest, C. R., et al. (2009). Programming cells by multiplex genome engineering and accelerated evolution. Nature 460, 894-898. doi: 10.1038/nature08187

Wang, Y. H., Wei, K. Y., and Smolke, C. D. (2013). Synthetic biology: advancing the design of diverse genetic systems. Annu. Rev. Chem. Biomol. Eng. 4, 69-102. doi: 10.1146/annurev-chembioeng-061312-103351

Warner, J. R., Reeder, P. J., Karimpour-Fard, A., Woodruff, L. B., and Gill, R. T. (2010). Rapid profiling of a microbial genome using mixtures of barcoded oligonucleotides. Nat. Biotechnol. 28, 856-862. doi: 10.1038/nbt.1653

Wendt, K. E., Ungerer, J., Cobb, R. E., Zhao, H., and Pakrasi, H. B. (2016). CRISPR/Cas9 mediated targeted mutagenesis of the fast growing cyanobacterium Synechococcus elongatus UTEX 2973. Microb. Cell Fact. 15:115. doi: 10.1186/s12934-016-0514-7

Wolk, C. P., Vonshak, A., Kehoe, P., and Elhai, J. (1984). Construction of shuttle vectors capable of conjugative transfer from Escherichia coli to nitrogenfixing filamentous cyanobacteria. Proc. Natl. Acad. Sci. U.S.A. 81, 1561-1565. doi: 10.1073/pnas.81.5.1561

Woo, H. M. (2017). Solar-to-chemical and solar-to-fuel production from CO2 by metabolically engineered microorganisms. Curr. Opin. Biotechnol. 45, 1-7. doi: 10.1016/j.copbio.2016.11.017

Xiao, Y., Wang, S., Rommelfanger, S., Balassy, A., Barba-Ostria, C., Gu, P., et al. (2018). Developing a Cas9-based tool to engineer native plasmids in Synechocystis sp. PCC 6803. Biotechnol. Bioeng. 115, 2305-2314. doi: 10.1002/bit.26747

Xiong, W., Cano, M., Wang, B., Douchi, D., and Yu, J. (2017). The plasticity of cyanobacterial carbon metabolism. Curr. Opin. Chem. Biol. 41, 12-19. doi: 10.1016/j.cbpa.2017.09.004 
Xiong, W., Morgan, J. A., Ungerer, J., Wang, B., Maness, P. C., and Yu, J. (2015). The plasticity of cyanobacterial metabolism supports direct $\mathrm{CO}_{2}$ conversion to ethylene. Nat. Plants 1:15053. doi: 10.1038/nplants.2015.53

Yang, Q., Pando, B. F., Dong, G., Golden, S. S., and van Oudenaarden, A. (2010). Circadian gating of the cell cycle revealed in single cyanobacterial cells. Science 327, 1522-1526. doi: 10.1126/science.1181759

Yokoo, R., Hood, R. D., and Savage, D. F. (2015). Live-cell imaging of cyanobacteria. Photosyn. Res. 126, 33-46. doi: 10.1007/s11120-0140049-x

Yoshikawa, K., Aikawa, S., Kojima, Y., Toya, Y., Furusawa, C., Kondo, A., et al. (2015). Construction of a genome-scale metabolic model of Arthrospira platensis NIES-39 and metabolic design for cyanobacterial bioproduction. PLoS ONE 10:e0144430. doi: 10.1371/journal.pone.0144430

Yoshikawa, K., Kojima, Y., Nakajima, T., Furusawa, C., Hirasawa, T., and Shimizu, H. (2011). Reconstruction and verification of a genome-scale metabolic model for Synechocystis sp. PCC 6803. Appl. Microbiol. Biotechnol. 92, 347-358. doi: 10.1007/s00253-011-3559-X

Yoshikawa, K., Toya, Y., and Shimizu, H. (2017). Metabolic engineering of Synechocystis sp. PCC 6803 for enhanced ethanol production based on flux balance analysis. Bioprocess Biosyst. Eng. 40, 791-796. doi: 10.1007/s00449-017-1744-8

Yu, J., Liberton, M., Cliften, P. F., Head, R. D., Jacobs, J. M., Smith, R. D., et al. (2015). Synechococcus elongatus UTEX 2973, a fast growing cyanobacterial chassis for biosynthesis using light and $\mathrm{CO}_{2}$. Sci. Rep. 5:8132. doi: $10.1038 /$ srep08132

Yu, Y., You, L., Liu, D., Hollinshead, W., Tang, Y. J., and Zhang, F. (2013). Development of Synechocystis sp. PCC 6803 as a phototrophic cell factory. Mar. Drugs 11, 2894-2916. doi: 10.3390/md11082894

Zang, X., Liu, B., Liu, S., Arunakumara, K. K., and Zhang, X. (2007). Optimum conditions for transformation of Synechocystis sp. PCC 6803. J. Microbiol. 45, 241-245.
Zavrel, T., Knoop, H., Steuer, R., Jones, P. R., Cerveny, J., and Trtílek, M. (2016). A quantitative evaluation of ethylene production in the recombinant cyanobacterium Synechocystis sp. PCC 6803 harboring the ethylene-forming enzyme by membrane inlet mass spectrometry. Bioresour. Technol. 202, 142-151. doi: 10.1016/j.biortech.2015. 11.062

Zhang, A., Carroll, A. L., and Atsumi, S. (2017). Carbon recycling by cyanobacteria: improving $\mathrm{CO}_{2}$ fixation through chemical production. FEMS Microbiol. Lett. 364, 1-7. doi: 10.1093/femsle/fnx165

Zhang, W., and Song, X. (2018). Synthetic Biology of Cyanobacteria. Singapore: Springer.

Zhang, Y. H. P. (2011). "Hydrogen production from carbohydrates: a mini-review," in Sustainable Production of Fuels, Chemicals, and Fibers from Forest Biomass, eds J. Zhu, X. Zhang, and X. Pan (Washington, DC: American Chemical Society), 203-216.

Zhou, J., Zhang, H., Meng, H., Zhu, Y., Bao, G., Zhang, Y., et al. (2014). Discovery of a super-strong promoter enables efficient production of heterologous proteins in cyanobacteria. Sci. Rep. 4:4500. doi: 10.1038/ srep 04500

Conflict of Interest Statement: The authors declare that the research was conducted in the absence of any commercial or financial relationships that could be construed as a potential conflict of interest.

Copyright (c) 2019 Santos-Merino, Singh and Ducat. This is an open-access article distributed under the terms of the Creative Commons Attribution License (CC BY). The use, distribution or reproduction in other forums is permitted, provided the original author(s) and the copyright owner(s) are credited and that the original publication in this journal is cited, in accordance with accepted academic practice. No use, distribution or reproduction is permitted which does not comply with these terms. 